\title{
The Intestinal Microbiome Primes Host Innate Immunity Against Enteric Virus Systemic Infection Through Type I Interferon
}

\section{Xiao-Lian Yang}

Key Laboratory of Animal Virology of Ministry of Agriculture, Center for Veterinary Sciences, Zhejiang University, Hangzhou

\section{Gan Wang}

Key Laboratory of Animal Virology of Ministry of Agriculture, Center for Veterinary Sciences, Zhejiang University, Hangzhou

\section{Jin-Yan Xie}

Key Laboratory of Animal Virology of Ministry of Agriculture, Center for Veterinary Sciences, Zhejiang University, Hangzhou

\section{Han Li}

Key Laboratory of Animal Virology of Ministry of Agriculture, Center for Veterinary Sciences, Zhejiang Universit, Hangzhou

Wei Liu

State Key Laboratory of Pathogen and Biosecurity, Beijing Institute of Microbiology and Epidemiology, Beijing

\section{Shu Jeffrey Zhu ( $\nabla$ shuzhu@zju.edu.cn )}

Zhejiang University https://orcid.org/0000-0003-2919-5473

\section{Research}

Keywords: microbiota, B. coccoides, macrophage, type I interferon, enteric virus

Posted Date: January 13th, 2021

DOl: https://doi.org/10.21203/rs.3.rs-142736/v1

License: (c) (i) This work is licensed under a Creative Commons Attribution 4.0 International License. Read Full License

Version of Record: A version of this preprint was published at mBio on May 11th, 2021. See the published version at https://doi.org/10.1128/mBio.00366-21. 
1 The intestinal microbiome primes host innate immunity against enteric virus

2 systemic infection through type I interferon

3 Xiao-Lian Yang ${ }^{1 \#}$, Gan Wang ${ }^{1 \#}$, Jin-Yan Xie ${ }^{1}$, Han Li ${ }^{1}$, Wei Liu ${ }^{3}$, and Shu J. Zhu ${ }^{1,2 *}$

$4 \quad$ \#Xiao-Lian Yang and Gan Wang contributed equally to this work.

$5 \quad{ }^{*}$ Correspondence: shuzhu@zju.edu.cn

$6 \quad{ }^{1}$ Key Laboratory of Animal Virology of Ministry of Agriculture, Center for Veterinary

$7 \quad$ Sciences, Zhejiang University, Hangzhou, P. R. China

$8 \quad{ }^{2}$ Department of Critical Care Medicine, Sir Run Run Shaw Hospital, Zhejiang University

9 School of Medicine, Hangzhou, P. R. China

$10{ }^{3}$ State Key Laboratory of Pathogen and Biosecurity, Beijing Institute of Microbiology and

11 Epidemiology, Beijing 100071, P. R. China

\section{Abstract}

Background: Intestinal microbiomes are of vital importance in antagonizing systemic viral infection. However, very little literature has shown whether commensal bacteria play a crucial role in protecting enteric virus systemic infection from the aspect of modulating host innate immunity. Also, only a few specific commensal bacteria species have been revealed to be capable in regulating antiviral innate immune responses mediated by type I interferon (IFN). The underlying mechanisms have not yet been elucidated.

Results: We utilized an enteric virus, encephalomyocarditis virus (EMCV) to inoculate PBStreated or antibiotic cocktail-administrated mice (Abx) orally or intraperitoneally to examine the impact of microbiota depletion on virulence and viral replication in vivo. Microbiota 
depletion exacerbated the mortality, neuropathogenesis, viremia and viral burden in brain following EMCV infection. Furthermore, Abx-treated mice exhibited severely diminished macrophage activation and impaired type I IFN production and ISG expression in PBMC, spleen or brain. With the help of fecal bacterial $16 \mathrm{~S}$ rRNA sequencing of PBS and Abx mice, we identified a single commensal bacterium Blautia coccoides (B. coccoides) that can restore macrophage- and IFNAR-dependent type I IFN responses to restrict systemic enteric virus infection.

Conclusion: Our present study demonstrates that intestinal microbiome is fundamental for protecting from enteric virus systemic infection through activating macrophages and type I IFN responses. Reconstitution with $B$. coccoides can inhibit enteric virus infection and mitigate its neuropathogenesis by activating IFN-I and ISG responses in macrophages via IFNAR- and STAT1-mediated signaling pathway.

Keywords: microbiota, B. coccoides, macrophage, type I interferon, enteric virus

\section{Introduction}

Mammalian intestines are colonized by trillions of microorganisms composed of bacteria, viruses, archaea and fungi, collectively referred to as intestinal microbiota [1, 2]. The widely diverse intestinal microbial communities have established an immensely complicated ecosystem that is of vital importance in maintaining host homeostasis. Cumulative evidence strongly supports the view that the dynamic crosstalk between the host and its indigenous commensal bacteria is fundamental for the development, induction, education and tuning of the host immune system [3]. As such, perturbation of the microbiota 
composition, (termed as 'dysbiosis') is linked to a myriad of metabolic and inflammatory diseases both at the intestinal mucosal sites and outside the gastrointestinal tract $[2,4,5]$. Over the past decade, rapid and extensive advances have shed light on the nature and utmost importance of intestinal microbes in regulating virus replication, transmission and pathogenesis. Multiple studies utilizing enteric viruses including poliovirus, retrovirus and noroviruses have demonstrated that the intestinal bacteria can promote viral infection [68]. The stimulating mechanisms include direct facilitation of viral binding to target cells, virion stabilization and indirect regulatory pathways of suppressing the mucosal immune responses $[7,9,10]$. However, as every coin has two sides, the signals form the indigenous microbiota are shown to be essential to protect the Drosophila from enteric virus oral infection by priming the antiviral innate immunity [11].

Other than exhibiting regulatory effect at mucosal sites during enteric viral infection, the gut microbiota has been shown to be essential in limiting viral systemic infection by calibrating innate immune responses mediated by mononuclear phagocytes [12-14]. A role for the commensal bacteria to regulate the systemic type I interferon (IFN-I) has been described in these studies, in which impaired production of IFN-I and interferon-stimulated genes (ISGs) were observed in antibiotic (Abx)-treated or germ-free (GF) mice infected with influenza A virus, murine cytomegalovirus, or Sendai virus, showing increased mortality and susceptibility $[13,15,16]$. Recently, two compelling back-to-back research articles reported that plasmacytoid dendritic cells ( $p D C s$ ) are the main cellular sources of Microbiota-induced IFN-I. Schaupp and colleagues demonstrated that microbiota-driven IFN-I expression by pDCs primes conventional dendric cells (cDCs) to initiate immune 
responses following pathogen encounter [17]. Meanwhile, Diamond's group has pointed out that IFN-I produced by microbiota-enabled pDCs prevents alphavirus, Chinungunya virus (CHIKV) in particular, from infection and dissemination in host blood monocytes [18]. The latter study has demonstrated that a single commensal bacterium, Clostridium scindens (C. scindens) can modulate prompt IFN-I responses through Toll-like receptor 7 (TLR7) and MyD88 signaling in pDC via primary to secondary bile acid (BA) transformation [18]. Although these studies provided great insights into the systemic effect of microbiota to limit viral infection, identification of unknown specific microbiome species that influence type I IFN antiviral responses is still lacking and the molecular links between the gut microbiota and type I IFN-meditated innate immunity are only beginning to be elucidated. Here, we described the impact of the intestinal microbiome on host IFN-I associated antiviral innate immunity using EMCV, a well-studied picornavirus that targets the central nerves system and is transmitted via fecal-oral route. Depletion of the gut microbiota with an Abx cocktail via oral gavage exacerbated the mortality and neurological symptoms to EMCV infection in mice. EMCV infection of Abx-treated mice had increased viral loads in blood and brain tissues. Coincidental with these phenotypes, the Abx-treated mice exhibited diminished innate immune responses reflected by impacted NK cell activity and macrophage activation post EMCV infection, and this was correlated with diminished expression of ISGs in the periphery and brain tissues. We performed the bacterial $16 \mathrm{~S}$ rRNA sequencing of the fecal samples collected from the Abx cocktails-treated or single Abx-treated mice to identify the possible commensal bacteria that may play important role in priming IFN-I expression. Monocolonization of Abx-treated mice with B. coccoides 
enabled them to regain the capability of restricting EMCV systemic infection by activating NK cells and macrophages through type I interferon expression. Thus, in the present study, we have identified a commensal bacterium that as far as we know, has not been previously documented to be able to regulate type I IFN-mediated innate immunity in the context of enteric virus systemic infection.

\section{Results}

\section{Microbiota depletion alters the neuropathogenesis and mortality to EMCV infection}

Groups of wild-type C57BL/6J mice (referred to as WT B6) were oral gavaged with a broadspectrum Abx cocktail, composed of Vancomycin (Van), Neomycin (Neo), ampicillin (Amp) and Metronidazole (Metro) consecutively for 5 days and infected orally with different doses of EMCV while the same Abx were kept in the water until the end of the experiments post inoculation $[6,19,20]$. The clinical symptoms and mortality rate of different groups was observed and documented for 14 days post-infection (dpi). As shown in Figure $1 \mathrm{~A}, 60 \%$ of Abx-treated mice inoculated with high dose of $2 \times 10^{7} \mathrm{TCID}_{50}$ units succumbed to infection by $9 \mathrm{dpi}$, only about $10 \%$ of Abx-treated mice succumbed at the dose of $2 \times 10^{5} \mathrm{TCID}_{50}$ units and no mortality occurred at the low dose of $2 \times 10^{3} \mathrm{TCID}_{50}$ units. However, all PBS-treated control survived without clinical symptoms during the entire observation period. Alongside the lethality phenotype, the Abx-treated mice infected with $2 \times 10^{7} \mathrm{TCID}_{50}$ units of EMCV developed various clinical symptoms including hunch and trembling, hind limb paralysis, dyspnea and death at $5 \mathrm{dpi}$, while the PBS-treated mice exhibited no obvious clinical signs (Fig. 1A). We then harvested the blood and brain tissues of the infected mice at 3 and 5 
111 dpi for virus loads detection using real-time RT-PCR. It was shown that EMCV per oral

112 inoculation resulted in significantly higher viral burden in the blood and brain tissues of

113 Abx-treated mice than the PBS-treated mice, indicating that viral replication in the

114 circulatory system and the target tissues was controlled by the presence of microbiota

115 during EMCV acute infection via oral delivery (Fig. 1B). Consistent with the clinical

116 symptoms and viral burden, Immunofluorescent assay revealed that there were more viral

117 signals (minor capsid protein VP2, Magenta) in the brain sections of Abx-treated mice than

118 the PBS-treated mice at 5 dpi (Fig. 1C, right panel). It seems that alteration of microbiota

119 composition does not alter the cellular tropism of EMCV in the brain for the majority of viral

120 signals were still co-stained with the marker of astrocytes (GFAP, red), but not with the

121 marker of microglial (IBA-1, green) in either PBS- or Abx-infected mice. Collectively, these

122 data indicate that the intestinal microbes are essential for limiting EMCV replication in the

123 target cells in the brain, and are required to protect the neurological diseases and cerebral

124 tissue lesions from EMCV oral infection.

125 To further confirm that the intestinal microbiome had an extraintestinal effect on limiting

126 EMCV systemic infection, we next infected both PBS- and Abx-treated mice systemically

127 via intraperitoneal injection to analyze the viral neurovirulence through this infectious route.

128 Indeed, Abx-treated mice were more susceptible to intraperitoneal infection with 200

$129 \mathrm{TCID}_{50}$ units of EMCV than PBS-treated animals and reintroduction of fecal bacteria into

130 Abx-treated animals alleviated EMCV mortality and disease (Fig. 1D). As the onset of viral

131 dissemination and in vivo replication is faster in the context of intraperitoneal inoculation

132 than per oral infection, we measured the viral loads in the blood and brain tissues earlier 
at 1 and $3 \mathrm{dpi}$ and discovered that the Abx-treated mice supported more significant EMCV replication than PBS-treated mice, whereas viral titers of Abx mice with fecal microbiota transplantation (FMT) were similar to those of PBS-treated mice (Fig. 1E). It was demonstrated that EMCV viremia and replication in target tissues was greatly increased in the absence of microbiota and FMT could restore the capability of systemic viral clearance.

Taken together, these data indicate that commensal bacteria are critical in promoting host systemic immunity against viral infection beyond the intestinal mucosal barrier.

\section{Microbiota deficiency severely diminishes innate macrophage antiviral immune responses to EMCV systemic infection}

The impaired viral clearance in the blood and brain of Abx-treated animals after EMCV infection provoke the hypothesis that the early systemic innate immune response was crippled by microbiota depletion. Thus, we first evaluated the recruitment and activation of early responding innate immune cells post EMCV infection by infecting PBS or Abx mice intraperitoneally with $200 \mathrm{TCID}_{50}$ units of EMCV and extracting the peritoneal blood mononuclear cells (PBMCs) and splenocytes at 3 dpi. The cell extracts were stained with different antibodies specific for indicated cell types and quantified by flow cytometry. Compared with their perspective mock control mice, infected PBS mice exhibited a significantly increased percentage of inflammatory monocytes, NK cells and macrophages, whereas there was a comparable frequency of $\mathrm{pDC}$ and $\mathrm{CDC}$. In contrast with these data, it seems that EMCV infection did not activate any of these cell subsets whatsoever regarding cellular frequencies in infected- versus mock Abx mice (Fig. 2A and Fig. S1). Also, analysis of the adaptive immune cells including $T$ cells and B cells suggests that 
commensal bacteria depletion did not alter the frequencies of T/B lymphocytes in the context of EMCV infection (Fig. 2B).

Previous studies have shown that NK cell priming is dependent on cytokines expressed by IFN-I-stimulated mononuclear phagocytes including DCs and macrophages [13, 21]. Macrophages, but not DCs had decreased expression of surface molecules that are critical during the early responses to lymphocytic choriomeningitis virus (LCMV) or influenza virus infection under the condition of Abx oral treatment [15]. Consistent with these findings, we discovered that surface molecules reflecting macrophage activation, like MHC-I and CD80 were greatly augmented in the PBMC macrophages from infected PBS mice, but not in those cells from infected Abx mice at 3 dpi compared to mock animals (Fig. 2C and Fig. S2). To further determine the requirement for macrophages in the systemic antiviral innate immunity and verify the necessity of intestinal microbiome in the macrophage response to viral infection, we treated PBS or Abx mice systemically with clodronate liposomes to deplete macrophages and analyze survival kinetics post EMCV intraperitoneal inoculation. Depletion of macrophages was at least $90 \%$ effective as assessed by flow cytometry (Fig. S3). For the mice that received control reagent, Abx treatment still resulted in significantly higher death rate than PBS controls, however for the mice injected with clodronate liposomes, Abx treatment did not make the animals more susceptible to EMCV infection (Fig. 2D). In support of this phenotype, at $1 \mathrm{dpi}$, greater titers of EMCV were detected in the blood of PBS mice than Abx mice both treated with control reagent, and no difference was observed between PBS or Abx mice treated with clodronate liposomes (Fig. 2E). Colonized mice treated with clodronate liposomes had almost 100 -fold higher blood viral 
loads than control reagent-treated colonized mice, indicating that macrophages are absolutely important for the early control of EMCV systemic infection. Additionally, blood viral titer of commensal bacteria-depleted macrophage knockout mice was also significantly higher than Abx mice without macrophage depletion, suggesting that commensal bacteria are not only required for activating macrophages, but also important for mediating other innate antiviral immune factors (Fig. 2E).

Together, these data suggest that gut microbiota can regulate the activation of macrophages, while disrupting the bacterial composition by antibiotic administration results in a significant impact on the macrophage-dependent protective innate immunity to restrain viral systemic infection.

\section{Commensal bacteria depletion greatly impairs the systemic and cerebral IFN-I} response following EMCV infection

Because IFN-I is the key factor that contributes to the stimulation of macrophages, which subsequently prime NK cells for antigen encounter via cytokine expression, therefore, we hypothesized that the microbiota could regulate the IFN-I response in our model. To test whether the commensal bacteria altered the profile of IFN-I production at different timepoints post infection, we inoculated groups of Abx-treated WT mice and PBS control with 200 TCID $_{50}$ units of EMCV intraperitoneally, collected blood and spleen at indicated timepoints post-infection and probed for IFN- $\beta$ and ISGs by real-time PCR. As early as 12 hours post-infection (hpi), there was significantly upregulated expression of IFN- $\beta$ and ISGs including Isg15, Isg56, Oas1a and Mx1 in the PBMC extract (Fig. 3A) and spleen (Fig. 3B) of PBS-treated mice compared to Abx-treated mice, suggestive of an impaired 
innate immune response mediated by IFN-I. Consistent with the affected systemic IFN-I response post-infection, there was reduced expression of IFN- $\beta$ and associated antiviral defense genes like Isg15, Irf7, IIf9 and Stat1/2 in the brain of Abx mice versus PBS mice at 1 and $3 \mathrm{dpi}$, indicating that the antiviral innate immunity is impeded not only systemically, but also locally at the target site of dissemination (Fig. 3C). In contrast, no differences in expression of proinflammatory cytokine TNF- $\alpha$, type II interferon, immune regulatory cytokine IL-17 and IL-10 were observed in the brain tissues of PBS and Abx mice at $3 \mathrm{dpi}$, implying that the impaired antiviral immunity in microbiome deficient Abx mice was not associated with the production of these cytokines (Fig. 3D).

To further confirm that commensal bacteria-driven type I interferon response is critical in antiviral innate immunity, we examined the requirement for the IFN-I induction and amplification pathway in EMCV early control with or without antibiotic suppression. Irf3-- or Ifnar ${ }^{\text {- }}$ mice that lacking key mediators either in type I interferon upstream induction pathway or amplification loop respectively, were utilized to clarify the effects of microbiota on different phases of IFN-I production upon EMCV systemic infection. Despite a narrowing phenotype comparing PBS- and Abx-treated wild-type mice $(P=0.0359)$, the lethality rate discrepancy in Irf3-- mice was still above $30 \%(P=0.106)$. On the contrary, all Ifnar ${ }^{/-}$mice were succumbed to EMCV inoculation by $5 \mathrm{dpi}$, showing identical susceptibility to EMCV intraperitoneal infection regardless of PBS or antibiotics treatment (Fig. 3E). In consistent with the phenotype on survival kinetics, the dissimilarities between PBS and Abx wild-type mice in viremia (20-fold) and brain viral replication (10-fold) at 1 dpi narrowed to 6.3 -fold and 3-fold in Irf3-- mice, whereas the differences were completely lost in Ifnar ${ }^{/-}$mice (Fig. 
3G). Collectively, these findings indicate that Irf3-mediated IFN-I induction pathway was partially affected by Abx treatment, while intestinal microbiome-correlated IFN-I amplification pathway is absolutely essential for preventing viral systemic infection.

\section{B. coccoides monocolonization alleviates EMCV pathogenesis and restricts viral}

We next investigate whether specific commensal bacteria taxa are responsible for the protein against EMCV systemic infection. First, we tested this idea by looking at which antibiotic in the cocktail was required for the effect on IFN-I modulation and whether the phenotype could be narrowed down to a single antibiotic treatment, or to a certain kind of alteration in the intestinal microbiome per se. Groups of WT B6 mice were treated with

231 combination of four antibiotics or single antibiotic of Van, Neo, Amp or Metro, inoculated

232 intraperitoneally with EMCV and the brain tissues were dissected at 3 dpi for EMCV viral burden measurement and IFN- $\beta$ expression detection. Generally, single Abx treatment significantly increased viral replication in the brain compared to PBS-treated controls except Metro or Neo. Van treatment conferred partial suppressive effect to EMCV early control as brain viral titers of these mice were not as high as Abx cocktail-treated animals. Amp was the most effective single antibiotic for the viral replication was comparable to Abx was not required for IFN- $\beta$ suppressive effect. Amp, Van or Neo treatment all dampened IFN- $\beta$ expression significantly compared to PBS mice. Among them, Amp treatment conferred an optimal suppression on IFN- $\beta$ expression level, Van treatment showed an 
intermediate effect, while Neo treatment had least impact on IFN- $\beta$ expression (Fig. 4B). As expected, successful inhibition of IFN- $\beta$ expression in brain tissues in general correlated with a substantial reduction in detectable $16 \mathrm{~S}$ rRNA readouts upon antibiotics treatment (Fig. 4C). In comparison, Van, Neo or Amp treatment had an effect is similar to that of the antibiotic cocktail whereas Metro had the least effective bacteria depletion (Fig.

4C). Based on these data, one would hypothesize that certain bacteria communities in the PBS control and resistant to Metro treatment, but significantly low in the Neo, Van, Amp or Abx mice, mediated the protective effect to EMCV systemic infection as an IFN-I inducer. Thus, we then carried out $16 \mathrm{~S}$ rRNA gene sequencing and analysis on fecal samples collected from PBS-, Abx cocktail- and single antibiotic-treated mice at 0 and $3 \mathrm{dpi}$ (Fig. 4D). With the hypothesis mentioned above, we chose three bacterial taxa of Blautia, Akkermansia and Lactobacillus because they had similar abundance between PBS- and Metro- treated mice at 0 and $3 \mathrm{dpi}$ in general (Fig. 4E). To explore whether they are the specific bacteria that mediated the protective effect of microbiota against viral systemic infection, we gavaged Abx mice with bacterial strain of $B$. coccoides (herein referred to as B.C), Akkermansia muciniphila (A. muciniphila, herein referred to as AKK), Lactobacillus reuteri (L. reuteri, herein referred to as L.R), an unrelated gram-positive human symbiont, Clostridium butyricum (C. butyricum, herein referred to as C.B), or mouse fecal contents (FMT) prior to EMCV intraperitoneal inoculation. Strikingly, colonization of Abx mice with a single bacterial strain B.C fully rescued EMCV mortality to the extent of FMT mice and that of PBS-infected controls (Fig. 4F), whereas gavage with C.B, L.R or AKK did not alter mortality, even though these bacteria strains exhibited efficient colonization (Fig. S4). 
Similarly, we observed significantly lower viral burden in the brain tissues of B.C colonized Abx mice in comparison to that of Abx, FMT or C.B-colonized Abx mice at 3 dpi (Figure 4G). Taken together, these data suggest that specific commensal bacteria $B$. coccoides protects host from EMCV systemic infection by restricting viral replication.

B. coccoides monocolonization promotes macrophage activation and IFN-I responses to systemic EMCV infection macrophage activation, we then gavaged Abx mice with B.C, C.B or fecal contents to evaluate expression of activation surface markers of MHC-I and CD80 in macrophages of PBMC and spleen. Indeed, B.C colonization in Abx mice remarkably increased the

275 frequency of MHC-I macrophages by $45 \%$ in PBMC (7.2\% to $52 \%)$ and by $13 \%$ in spleen (from $13.5 \%$ to $26 \%$ ) macrophages following EMCV infection at 3 dpi, comparable to FMT mice and PBS controls (Fig. 5A). In a similar way, CD80-upregulated macrophages were elevated from $10 \%$ to $26.4 \%$ in PBMC, and from $7.36 \%$ to $53.7 \%$ in spleen macrophages post B.C colonization in the context of EMCV infection (Fig. 5B). However, unlike B.C colonized-Abx mice exhibited completely recovered MHC-I upregulation in both PBMC and spleen macrophages to FMT treatment and PBS controls, the CD80 upregulation was only partially rescued by B.C monocolonization especially in PBMC macrophages that the magnitude of increase was significantly smaller than FMT mice (Fig. 4B, 16.4\% versus $37.5 \%$ ), indicating that the expression of this surface molecule might not be only regulated by $B$. coccoides, but is dependent on other intestinal bacteria as well. Likewise, C.B

286 colonized-mice remained low level of either MHC-I- or CD80-upregulated PBMC and 
spleen macrophages compared to Abx mice following viral infection at $3 \mathrm{dpi}$. To determine whether B. coccoides mediates protection from EMCV systemic infection through a macrophage-dependent mechanism, we then colonized macrophage-depleted Abx mice with $B$. coccoides before EMCV intraperitoneal inoculation and observed the survival curves for 14 days. It was shown that clodronate treatment abolished B.C-mediated protection, demonstrating that B.C is required for conferring macrophage-mediated 293 protection against EMCV infection (Fig. 5C).

To determine whether colonizing Abx mice with B. coccoides restores systemic IFN-I responses to viral infection, we harvested PBMCs from Abx mice with B.C, C.B or fecal contents colonization at $3 \mathrm{dpi}$ and subjected to IFN- $\beta$ expression measurement by qPCR. Apparently, B.C colonization in Abx-treated mice recovered IFN- $\beta$ expression in PBMCs to the level of that detected in FMT and PBS-treated controls upon EMCV infection at 3 dpi, whereas C.B colonization did not (Fig. 5D). Since we have demonstrated that intestinal microbiome-driven IFN-I amplification pathway is absolutely essential for antagonizing viral EMCV infection in an IFN-I dependent manner. We colonized Ifnar/- mice with B.C prior to virus inoculation and discovered that these bacterium colonized-mice did not display less comparison to Abx Ifnar ${ }^{-}$controls at $1 \mathrm{dpi}(\mathrm{Fig} .5 \mathrm{~F})$. As a whole, these data demonstrate that the protective effect of $B$. coccoides against viral systemic infection required macrophages and IFN-I amplification signaling. 

EMCV infection

311 To directly clarify whether B. coccoides can promote systemic type I IFN responses through

312 macrophages activation, we infected bone-marrow-derived macrophages (BMDMs)

313 isolated from PBS-, Abx-treated, B.C-colonized, or C.B colonized mice with EMCV at MOI

314 of 5 in vitro and detected induction of Ifnb and associated ISGs at 8 hpi using qRT-PCR.

315 Expression of Ifnb and ISGs including Oas1a, Isg15 and Mx1 was drastically reduced in

316 BMDMs isolated from Abx mice compared to PBS mice, suggestive of an intrinsic

317 incapability of responding to viral infection, but this responsiveness was partially regained

318 by B.C, not C.B colonization (Fig. 6A). Furthermore, B.C colonization in Abx mice endowed

319 their BMDMs with the ability to induce ISGs expression in an Ifnar-dependent manner for

320 ISGs expression was undetectable (or extremely low) in BMDMs isolated from B.C-

321 colonized Ifnar ${ }^{\text {- }}$ mice following EMCV infection at 8 hpi (Fig. 6B). As STAT1 conveys

322 signals downstream of IFN receptor engagement and its phosphorylation and nuclear-

323 translocation mediates transcription of ISGs in the IFN-I amplification loop, thus we

324 stimulated macrophages isolated from spleen of PBS, Abx or B.C colonized-Abx mice with

325 IFN- $\gamma$ in vitro and tested STAT1 phosphorylation using flow. IFN- $\gamma$-stimulated

326 macrophages isolated from Abx mice exhibited significantly low level of pSTAT1 compared

327 to those from PBS mice, while B.C colonization in Abx mice resulted in recovered STAT1

328 phosphorylation in splenic macrophages comparable to PBS controls (Fig. 6C and Fig. S5).

329 We also looked at expression of multiple antiviral defense genes expression in

330 macrophages isolated from the spleen of infected PBS, Abx, B.C-colonized animals to 
confirm whether same phenotype exists in vivo post viral infection. At $1 \mathrm{dpi}$, splenic macrophages from Abx mice exhibited significantly decreased antiviral gene expression compared to those from PBS mice, but B.C colonization in Abx mice induced significantly higher level of Ifnb and ISGs, although not fully stored to the level of PBS controls (Fig. 6D). This phenotype is consistent with the data showing B.C colonization partially restored the frequency of CD80-expressing macrophages (Fig. 5C) in PBMC, implying other commensal bacteria species also possess the capability of inducing type I IFN and ISG responses in circulating macrophages. Again, B.C exerts its effects through IFNARmediated IFN-I signaling because no detectable or obviously upregulated antiviral gene expression was observed in macrophages sorted from spleen of B.C colonized Abx Ifnar ${ }^{/-}$ mice (Fig. 6E). Overall, these findings suggest that $B$. coccoides is sufficient for maintaining optimal macrophage responsiveness to stimuli (cytokine and viral infection) and it is very likely that $B$. coccoides augments type I IFN signaling in macrophages via IFNAR and STAT1 phosphorylation to protect host from virus systemic infection.

\section{Discussion}

In the present study, we have described a crucial role for the commensal microbiota in modulating antiviral innate immunity towards enteric virus systemic infection. In particular, the intestinal microbiome protects systemic viral infection by promoting type I IFN responses in peripheral innate immune cells, mainly macrophages, via an Ifnar-dependent signaling pathway. Furthermore, we have identified the mechanism by which an understudied commensal bacteria species, $B$. coccoides, utilizes to restrict enteric virus systemic 
infection through enhancing type I IFN responses in macrophages.

Over the past ten years, it has been gradually acknowledged that the intestinal microbiome can shape host antiviral immunity. Accumulating literature has shown that animals administered with cocktails of broad-spectrum antibiotics or GF mice have impaired innate and adaptive immune responses following infections by various viral pathogens including LCMV, influenza virus, murine cytomegalovirus (MCMV), flaviviruses, VSV or CHIKV [13, $15,16,18,22]$. However, up till now, all studies on mammalian enteric viruses have reported that intestinal microbiome promotes virus infection $[6,7,10,20,23]$, with two exceptions. One conducted by Grau et al reported that C. scindens primes type III interferon induction to suppress murine norovirus infection in proximal small intestines by bile acids biotransformation[19]. Another example of commensal bacteria-mediated antienteric virus protection was provided by Shi et al, demonstrating that segmented filamentous bacteria (SFB) prevent and cure rotavirus infection by accelerating epithelial cell turnover [24]. Unlike norovirus and rotavirus infection only causes self-limited diseases, some enteric viruses, enterovirus 71 and EMCV for instance, can result in systemic infection and lead to a diverse array of neurological disease $[25,26]$. To answer the question whether intestinal microbiota could be protective indirectly by regulating host antiviral innate immunity during enteric virus infection, we infected wild-type mice with

371 EMCV per orally to mimic its natural infection, or through intraperitoneal route to 372 immediately establish systemic infection. To our surprise, microbiota depletion with antibiotics exacerbates the neurological disease and enhances viral replication upon

374 EMCV infection under both routes of inoculation (Fig.1). This phenotype resembles the 
results reported by previous studies on non-enteric viral pathogens mentioned above, but not similar to the milestone study conducted by Kuss et al using poliovirus and reovirus, in which intestinal microbiomes and LPS are reported to promote viral replication and systemic pathogenesis [6]. These discrepancies suggest that microbiota acts very differently in interacting with even two closely related enteric viruses (EMCV and poliovirus are both picornaviruses that share very similar viral structure). It is highly likely that the innate immune responses were also severely diminished by antibiotics treatment in their model (we used the same antibiotic regimens) whereas poliovirus replication in vivo was barely established to reflect the defects on host innate immunity in the absence of microbiota. Conversely, EMCV in vivo replication and dissemination does not require microbiota in our model (Fig. 1B and 1C) so that the neuropathogenesis was deteriorated in the context of antiviral innate immunity being dampened by microbiota depletion.

Collectively, multiple studies have demonstrated that commensal bacteria are responsible for shaping host antiviral immunity beyond mucosal sites. GF mice and Abx-treated mice exhibited blunted type I interferon responses that are required for optimizing functions of nonmucosal immune cells including NK cells, macrophages, or CD8 ${ }^{+} \mathrm{T}$ cells $[13,14,16$, 22]. In our study, we showed that macrophages isolated from Abx mice did not display higher frequency or upregulated expression of activating surface markers like MHC-I or CD80 at $3 \mathrm{dpi}$ (Fig. 2A and 2D). Additionally, macrophage depletion equalized the discrepancies in mortality and viral replication between Abx-treated mice and PBS controls, indicating that macrophages are essential for microbiota-mediated protection from EMCV systemic infection (Fig. 2E and $2 \mathrm{~F}$ ). Our data are in consistent with previous studies 
demonstrating that microbiome can regulate antiviral macrophage responses by inducing the expression of type I IFN and ISG genes after influenza virus infection $[15,16]$. Similar to these studies, we also showed that microbiota depletion results in severely diminished unrestricted virus replication and exacerbates neuropathogenesis and mortality. The type I IFN and ISG responses both in periphery and brain (Fig. 3A-D), which causes impaired IFN-I production and ISG expression in the brain tissues of Abx mice suggests major defects in responsiveness of microglia to viral infection because they are the main sources of IFN production in brain. The results are consistent with one manuscript reporting that microglia of GF mice were unable to produce various cytokines and chemokines upon stimulation[27]. Our model figured out that the microbiota-driven IFN and ISG responses are partially dependent on Irf3-mediated IFN-I induction and are fully relied on Ifnarmediated signaling pathway (Fig. 3E and 3F).

Although accumlated evidences has shown that microbiota can promote ISG responses in multiple cell types, the cellular sources of type I IFN are yet less-well defined until very recently, where two papers demonstrated that microbiota constitutively induces type I IFN production and basal level of ISG expression in pDCs at systemic sites [17, 18]. In our study, we only looked at the frequency of pDCs in PBMC or spleen following EMCV infection at $3 \mathrm{dpi}$, and observed no differences between PBS and Abx mice (Fig. 2A). Similarly, Abt et al reported that at 3 dpi following influenza virus infection, there was a comparable influx of pDCs into bronchiole alveolar lavage and the pDCs exhibited similar activation profile in PBS and Abx mice [15]. These seemingly discordant observations could be explained by the timing of detection because pDCs respond to virus nucleic acids 
with massive and rapid secretion of IFN-I (1-3 h post stimulation) independently of the IFNAR-based feedback signaling that is always required for most cell types in IFN-I production [28]. Also, profiling the IFN-I and ISG expression in pDCs could be very subtle especially at steady state ( $0 \mathrm{dpi})$ and using different microbiota-deficient mouse model (GF versus Abx-treated) may result in distinct phenotype $[17,18]$. Generally, it is plausible that signals of commensal bacteria drive a very swift first wave of IFN-I (especially IFN- $\beta$ ) production in poised pDCs to activate NK cells, macrophages and overall innate immune responses to viral infection.

Up till now, only a few publications have begun to identify specific commensal bacterial species that play an important role in inducing type I IFN-mediated innate antiviral immunity $[16,18,29,30]$. Although Abt and colleagues' study revealed an interplay between commensal bacteria and poised low-level tonic antiviral interferon signaling, they have not figured out the specific bacterial species that regulates the 'steady-state' readiness of antiviral pathways in macrophages [15]. Our work builds on the limited existing literature by identifying a less documented-commensal bacterium, $B$. coccoides that plays a crucial role in protecting from enteric virus systemic infection through IFN-I induction. Although the genus of Blautia only comprises less than $4 \%$ of total colonic microbes (Fig. $4 \mathrm{E}$ ), this bacterial community seems to be highly capable of activating macrophages as $B$. coccoides colonialization almost fully recovered the upregulation of MHC-I and CD80 on the surface of PBMC and spleen macrophages in Abx-treated animals following EMCV infection (Fig. 5B and 5C). Single colonization of $B$. coccoides in Abx mice can also rescue the intrinsic defects in expression of IFN-I and key ISGs in BMDMs in an Ifnar-dependent 
manner following EMCV infection in vitro (Fig. 6A and 6B), or restore the STAT1 phosphorylation in splenic macrophages following IFN- $\gamma$ stimulation in vitro (Fig. $6 \mathrm{C}$ ). The present study adds a "missing piece' to the previous work by demonstrating a single commensal bacteria strain $B$. coccoides can restore the impotency of macrophages in responsiveness to type II IFN or virus because of microbiota depletion.

Since B. coccoides monocolonization in Abx-treated mice restored protection of EMCV systemic infection by restricting in vivo viral replication in an IFNAR- and macrophagedependent manner (Fig. 4F, 4G; 5D and 5F), a next question will be what does $B$. coccoides utilize to induce IFN-I responses in macrophages to confer antiviral systemic protection? Two recent reports suggest that C.scindens-derived metabolite deoxycholic acid or glycolipids of B.fragilis can impact host antiviral innate immunity by inducing type I IFN production and ISG expression in pDCs or a subset of colonic cDCs [18, 30]. Short-chain fatty acids (SCFA), primarily produced by Gram-positive bacteria Firmicutes and Bacteroidetes through fermentation of undigested polysaccharides, are not only important local energy sources for gut microbiota and intestinal epithelial cells, but also crucial regulators for shaping immune systems at extraintestinal sites [5]. As the most abundant SCFA in the colon, nearly one-third of acetate is produced by acetogenic bacterial communities such as Blautia [31]. It was shown that acetate derived from high-fiber diet protects against respiratory syncytial virus infection by promoting type I IFN production and ISG expression in pulmonary epithelial cells through GPR43 [32]. Future studies will be carried out to examine the effects of specific SCFAs on influencing host anti-enteric virus innate immunity using microbiota deficient mouse model. 


\section{Conclusion}

465 Our present study demonstrates how fundamental innate immune mediators such as macrophages and type I IFN are regulated by commensal bacteria to antagonize enteric virus systemic infection. In particular, we have identified a novel commensal bacterial strain,

B. coccoides can restrict enteric virus replication and neuropathogenesis by activating IFNI and ISG responses in macrophages via IFNAR- and STAT1-mediated signaling pathway. This finding may provide insight into the development of novel therapeutics in preventing enteric virus infection or possibly, mitigating its clinical diseases.

\section{Methods}

\section{Cell, bacteria isolates and virus}

BHK-21 cells were cultured in DMEM supplemented with $10 \%$ fetal bovine serum (GIBCO ${ }^{\mathrm{TM}}$, Invitrogen Corporation, Carlsbad, $\mathrm{CA}, \mathrm{USA}$ ) at $37^{\circ} \mathrm{C} / 5 \% \mathrm{CO}_{2}$.

B. coccoides was purchased from ATCC (ATCC 29236) and cultured in Modified Chopped Meat Medium (ATCC Medium 1490, ELITE-MEDIA) at $37^{\circ} \mathrm{C}$ under anaerobic conditions. C. butyricum was purchased from ATCC (ATCC 19398) and cultured in Thiolglycollate Medium (Hopebio) at $37^{\circ} \mathrm{C}$ under anaerobic conditions. A. muciniphila was kindly provided by Dr. Lan-juan Li (The First Affiliated Hospital, College of Medicine, Zhejiang University, Hangzhou, P. R. China) and cultured in Brain Heart Infusion Medium (BHI, OXOID) at $37^{\circ} \mathrm{C}$ under anaerobic conditions. L. reuteri was purchased from China Center for Type Culture

484 Collection (CCTCC, AB 2014289) and cultured in DeMan-Rogosa-Sharpe medium 
(OXOID) at $37^{\circ} \mathrm{C}$ under anaerobic conditions. The concentration of each bacterial species was quantified based on CD600.

Recombinant virus derived from full-length clone of EMCV strain BJC3 (GenBank: DQ464062) was used in this study [33] and viral titers of EMCV stocks were determined by standard $\mathrm{TCI}_{50}$ assay described previously [34].

\section{Mice and infections}

Mice were maintained in specific pathogen-free (SPF) facility with temperature- and humidity-controlled environment $\left(22 \pm 2^{\circ} \mathrm{C}, 50 \pm 10 \%\right.$ humidity), and all animal experiments were strictly carried out in accordance with protocols approved (\#117113) by the Institutional Animal Care and Use Committee of Zhejiang University. Six- to eight-week-old, sex-matched mice were used for all experiments. The C57BL/6J wildtype mice were purchased from the Model Animal Research Center of Nanjing University (Nanjing, China). Type 1 interferon receptor knockout mice (referred to as $/$ fnar $^{-/-}$) were kindly gifted by Dr. Yu Chen (Wuhan University, Hubei, P. R. China) and interferon regulatory factor 3 deficient mice (referred to as $I \mathrm{rf}^{-/-}$) mice were provided by Dr. Jin Jin (Zhejiang University, P. R. China).

For all wild-type B6 mice studies, animals were inoculated orally with $2 \times 10^{7}, 2 \times 10^{5}$ or $2 \times 10^{3} \mathrm{TCID}_{50}$ units of EMCV in $25 \mu \mathrm{L}$ inoculum, or infected intraperitoneally with 200 $\mathrm{TCID}_{50}$ units of EMCV in $200 \mu \mathrm{L}$ inoculum. For EMCV virulence assay on Ifnar ${ }^{-/-}$, Irf3 ${ }^{-/-}$ mice or wild-type mice treated with clodronate liposomes, animals were inoculated intraperitoneally with $50 \mathrm{TCID}_{50}$ units of EMCV in $200 \mu \mathrm{L}$ inoculum. Clinical symptoms were observed and documented blindly using following scoring criteria: 0 , normal; 1 , 
hunch-back and trembling; 2, hind limb paralysis; 3, dyspnea and unresponsive to touch; 4 , sudden death.

For viral titer determination, tissue samples at indicated time points were harvested, weighed, and homogenized with stainless beads in $1 \mathrm{~mL}$ of DMEM media supplemented with $2 \%$ FBS and titrated by qPCR. Briefly, tissue samples were homogenized at $45 \mathrm{~Hz}$ for 1 minutes and the homogenates were clarified by centrifugation at 12,000 rpm for $5 \mathrm{~min}$. Total RNA was extracted with Trizol Reagent (Invitrogen) and subjected to quantitative reverse transcriptase PCR (qRT-PCR) using One-step qPCR Kits (TOYOBO) on an ABI 7500 Fast Instrument. Standard cycling conditions and primers/probe (forward: 5'TGAGTCATTAGCCATTTCAACCCA-3'; reverse: 5'-CGTGAGATACAAACCCGCCCTA-3'; probe: 5'-TCCCATCAGGTTGTGCAGCGA-3') were described previously [35]. Viral burden was expressed on a log10 scale as EMCV genomic RNA equivalents per milligram. colonization

Mice were administrated with antibiotic cocktail comprised of $10 \mathrm{mg}$ each of ampicillin, neomycin, metronidazole and vancomycin $(167 \mathrm{mg} / \mu \mathrm{L})$ daily for 5 days via oral gavage. After the fifth day of oral gavage, antibiotics were added to the drinking water at a

524 concentration of $1 \mathrm{~g} / \mathrm{L}$ for ampicillin, neomycin and metronidazole and $500 \mathrm{mg} / \mathrm{L}$ for 525 vancomycin. Fecal samples collected from microbiota-depleted mice at the 5 th day post 526 treatment were homogenized, plated on brain-heart infusion $(\mathrm{BHI})$ agar with $10 \%$ sheep blood and cultured under anaerobic conditions at $37^{\circ} \mathrm{C}$ for $2 \mathrm{~d}$ followed by aerobic conditions at $37^{\circ} \mathrm{C}$ for $1 \mathrm{~d}$ to confirm efficient microbial depletion. Animals were maintained 
with Abx- or PBS-containing water for the duration of the experiment [6, 20].

For FMT experiments, $200 \mathrm{mg}$ of pooled feces pellets were homogenized with sterile silica beads in $1.5 \mathrm{ml}$ PBS at $45 \mathrm{~Hz}$ for 1 minutes and filtered with $70 \mu \mathrm{m}$ strainers. Mice were administrated Abx as described above, Abx administration was discontinued on day 6 and Abx mice were gavaged with $150 \mu l$ filtered stool homogenates individually [16]. For bacterial colonization experiments, Abx mice were gavaged with $10^{10} \mathrm{cfu}$ of $B$. coccoides, A. muciniphila, C.butyrum or L. reuteri in $150 \mu \mathrm{l}$ PBS at the $6^{\text {th }}$ day post Abx oral administration. At 48 hours post FMT or bacterial colonization (on day 8 post Abx oral gavage), stool samples were collected to determine the efficiency of colonization and colonized-mice were inoculated intraperitoneally with EMCV at indicated doses.

\section{Immunofluorescent assay (IFA) and viral signal quantification}

Brain tissues were collected from either mock or infected mice at 3 or $5 \mathrm{dpi}$ and fixed in $4 \%$ buffered paraformaldehyde and embedded in paraffin. Deparaffinized brain sections were incubated with $10 \%$ normal goat serum for 30 min to block nonspecific binding, co-stained with anti-EMCV VP2 (1: 500) and anti-GFAP monoclonal antibodies (1: 1200, ABcam), or co-stained with anti-VP2 and anti-IBA1 monoclonal antibodies (1: 800, Invitrogen). Following primary antibodies incubation at $4^{\circ} \mathrm{C}$ overnight, a Cy3-conjugated anti-mouse secondary antibody at a dilution of 1:100 (Santa Cruz Biotechnology, Santa Cruz) was added to the sections and incubated for $30 \mathrm{~min}$ at room temperature. Stained sections were imaged with a NIKON ECLIPSE C1 Upright Fluorescent Microscope. The number of antigen-positive cells from 10 fields of view (200x) was combined when a single section 
mice [33].

\section{Splenocytes, bone marrow derived macrophage and peripheral blood mononuclear}

\section{cells generation and treatment}

Spleens were dissected and single cell suspensions of splenocytes were generated by grinding through a 70-mm strainer. Erythrocytes were lysed with Red Blood Cell Lysis Buffer (RCLB, HyClone), and remaining cells were resuspended in PBS supplemented with $2 \%$ FBS and 1 mM EDTA.

Bone marrow derived macrophages (BMDMs) were generated by isolation of bone marrow cells from mouse femurs and tibiae after sacrifice. Cells were cultured at $37^{\circ} \mathrm{C}$ in DMEM supplemented with $20 \%$ FBS and $30 \%$ supernatant of filtered-L929 cells for 7 days prior to experimental procedure. BMDMs were infected with EMCV at MOI 5 for 8 hours prior to harvest for indicated groups.

Peripheral blood mononuclear cells (PBMCs) isolation was performed by using density gradient centrifugation with a PBMC isolation kit (TBD).

\section{IFN- $\beta$ protein analysis}

IFN- $\beta$ protein quantification was performed using Mouse IFN-beta ELISA Kit (ABclonal) according to manufacturer's instructions.

\section{Cytokine expression analysis}

RNA was isolated from tissues or cells using Trizol Reagent (Invitrogen) as per manufacturer's instructions. Gene expression levels of Ifnb, Isg15, Isg56, Irf7, Irf9, Stat1, Stat2, Oas1a, $M x 1$ and $C x c / 10$ (primers used for the assay were listed in Table. S1) were 
determined via qRT-PCR and normalized to GAPDH expression. Results are presented

573 as fold changes of cytokine expression in infected mice over mock animals $\left(2^{-\Delta \Delta C t}\right)$.

574

\section{Macrophages depletion}

Macrophages depletion experiments were performed as previously published literatures.

Briefly, animals were injected with 250 ul per mouse of clodronate liposomes (YEASEN) or control liposomes intraperitoneally 2 days prior to Abx treatment, the day of Abx treatment, and then on days 2 and 5 post Abx treatment.

\section{DNA extraction, 16S rRNA amplicon sequencing and data analyses}

Genomic DNA of the fecal samples was extracted using the ALFA-SEQ Advanced Stool DNA Kit (Magen). The quality and quantity of DNA were measured using a NanoDrop One (Thermo Fisher Scientific). Subsequently, Bar-coded PCR primers targeting V3-V4 region of bacterial 16S rRNA genes were used to generate amplicons (Forward: ACTCCTACGGGAGGCAGCA; Reverse: GGACTACHVGGGTWTCTAAT) and multiplex sequencing of amplicons with sample-specific barcodes was performed using an Illumina Novaseq 6000 platform (paired end $2 \times 250$ nucleotide reads, Guangdong Magigene Biotechnology Co., Ltd. Guangzhou, China).

The raw data of sequencing were filtered using the fastp (version 0.14.1, https://github.com/OpenGene/fastp) with parameters -W 4 -M 20 and further processed by the cutadapt (https://github.com/marcelm/cutadapt/) to remove the primer sequences to obtain the paired-end clean data. Subsequently, the usearch -fastq_mergepairs tool (version 10, http://www.drive5.com/usearch/) was utilized to merge the raw tags, which were later trimmed by the fastp to get the clean tags. The operational taxonomic units 
594 (OTUs) were then clustered with a cut-off value of $97 \%$ similarity using the UPARSE 595 software. Each representative sequence of these OTUs were assigned using the SILVA 596 database to annotate taxonomic information. The richness of certain commensal bacteria 597 taxa was calculated using the usearch -alpha_div (version 10, 598 http://www.drive5.com/usearch/) according to the OTU abundance. Based on the relative 599 abundance of species at each classification level in otu_table, $\mathrm{R}$ software was used to 600 draw the histogram, heat map and ternary phase diagram.

\section{Flow cytometry and cell sorting}

602 Splenocytes and PBMCs were harvested to analyze levels of different antigen on the 603 surface of different cell subsets following blockade of Fcy receptors with anti-CD16/32 604 (eBioscience). Fluorescently conjugated antibodies used include those specific to CD3, 605 CD4, CD8, CD19, CD11b, CD11c (Biolegend), Ly6c, F4/80, MHC II and PDCA-1 606 (eBioscience). Inflammatory monocytes were identified as Ly6c $\mathrm{c}^{+}$and CD11 b+ $\mathrm{b}^{+}$PBMC and 607 splenic macrophages were identified as F40/80 and CD11b double positive. NK cells were

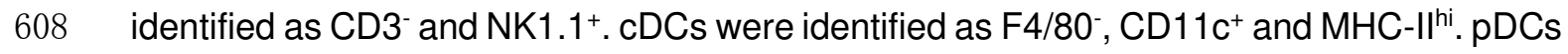
609 were identified as CD11 $\mathrm{c}^{\mathrm{int}}$ and PDCA-1+. CD4 T cells, CD8 T cells and B cells were 610 identified as $\mathrm{CD}^{+} / \mathrm{CD}^{+}, \mathrm{CD}^{+} / \mathrm{CD}^{+}$or $\mathrm{CD} 19^{+}$respectively. For Intracellular staining, cells 611 were permeabilized with Cytofix/Cytoperm buffer (BD), and stained for INF-y or granzym

612 B. Splenic macrophages were sorted as F40/80 and CD11b double positive subsets by

613 flow cytometry using the BD FACSVerse.

\section{In vitro phosflow STAT1 staining of macrophages}

615 Splenic macrophages were stimulated with $200 \mathrm{ng} / \mathrm{ml}$ recombinant IFN-ץ (R\&D Systems) 
for 16 hours, media was subsequently removed and replaced with $0.05 \%$ trypsin and incubated at $37^{\circ} \mathrm{C}$ for 2 min. Cells were then fixed with $4 \%$ PFA for 10 min, stained for surface markers, permeabilized with Cytofix/Cytoperm buffer, and stained for pSTAT1 with PE-conjugated anti-STAT1 (pY701) antibody (Biolegend).

\section{Statistical Analysis}

Statistical analyses were performed with Prism GraphPad software v 8.0. Error bars represent standard errors of mean in all figures and $P$ values were determined by unpaired, two tailed Student's t test. Log-rank test was used for survival curves. $\left({ }^{*} p<0.05 ;{ }^{* *} p<0.01\right.$; $\left.{ }^{* * *} p<0.001 ;{ }^{* * * *} p<0.0001\right)$

\section{List of abbreviations}

type I interferon (IFN), encephalomyocarditis virus (EMCV), antibiotic cocktailadministrated mice (Abx), Blautia coccoides (B. C), interferon-stimulated genes (ISGs), germ-free (GF), plasmacytoid dendritic cells (pDCs), conventional dendric cells (cDCs), Chinungunya virus (CHIKV), Clostridium scindens (C. scindens), Toll-like receptor 7 (TLR7), secondary bile acid (BA), Vancomycin (Van), Neomycin (Neo), ampicillin (Amp) and Metronidazole (Metro), days post-infection (dpi), ecal microbiota transplantation (FMT), peritoneal blood mononuclear cells (PBMCs), lymphocytic choriomeningitis virus (LCMV), hours post-infection (hpi), Akkermansia muciniphila (A. muciniphila, herein referred to as AKK), Lactobacillus reuteri (L. reuteri, herein referred to as L.R), Clostridium butyricum (C. butyricum, herein referred to as C.B), murine cytomegalovirus (MCMV), segmented filamentous bacteria (SFB) 
Not applicable.

642

Consent for publication

Not applicable.

645

Availability of data and materials

647

Not applicable.

648

Competing interests

650

The authors declare that they have no competing interests.

651

\section{Funding}

653 This research was funded by the National Key Research and Development Program of China (No. 2018YFD0500102) and supported by the Fundamental Research Funds for the Central Universities.

\section{Author's contributions}

Xiao-Lian Yang, Gan Wang, Jin-Yan Xie conceptualized the project. Shu J. Zhu, Gan Wang and Jin-Yan Xie wrote the paper. Wei Liu and Shu J. Zhu reviewed and edited the paper. 
Xiao-Lian Yang performed the virulence studies, viral loads titration, IFN-I and ISGs expression analysis, IFA, flow cytometry of immune cells and activation of macrophages.

Gan Wang performed bacteria colonization, pathogenesis studies, macrophage sorting and pSTAT1 staining, IFN-I and ISGs expression analysis. Jin-Yan Xie analyzed and interpreted 16S rRNA sequencing data. Han Li extracted DNA from fecal samples.

\section{Acknowledgments}

We appreciate Dr. Lan-juan Li for providing valuable bacterial strain Akkermansia muciniphila. We thank Dr. Jun Chen and Dr. Jin Jin for providing respective knockout mouse strains. We would also like to acknowledge Dr. Melissa K. Jones for helpful discussions and critical reviewing of the manuscript.

\section{References}

1. Qin, J., R. Li, J. Raes, M. Arumugam, K. S. Burgdorf, C. Manichanh, et al., A human gut microbial gene catalogue established by metagenomic sequencing. Nature, 2010. 464(7285): p. 59-65.

2. Blander, J. M., R. S. Longman, I. D. Iliev, G.F. Sonnenberg, and D. Artis, Regulation of inflammation by microbiota interactions with the host. Nat Immuno1, 2017. 18(8): p. 851-860.

3. Belkaid, Y. and 0. J. Harrison, Homeostatic Immunity and the Microbiota. Immunity, 2017. 46(4): p. 562-576.

4. Kau, A. L., P. P. Ahern, N. W. Griffin, A. L. Goodman, and J. I. Gordon, Human nutrition, the gut microbiome and the immune system. Nature, 2011. 474(7351): p. $327-36$.

5. Rooks, M. G. and W. S. Garrett, Gut microbiota, metabolites and host immunity. Nat Rev Immuno1, 2016. 16(6): p. 341-52.

6. Kuss, S. K., G. T. Best, C.A. Etheredge, A. J. Pruijssers, J. M. Frierson, L.V. Hooper, et al., Intestinal microbiota promote enteric virus replication and systemic pathogenesis. Science, 2011. 334(6053): p. 249-52.

7. Kane, M. , L. K. Case, K. Kopaskie, A. Kozlova, C. MacDearmid, A. V. Chervonsky, et al., Successful transmission of a retrovirus depends on the commensal 
microbiota. Science, 2011. 334(6053): p. 245-9.

8. Jones, M. K., K. R. Grau, V. Costantini, A. 0. Kolawole, M. de Graaf, P. Freiden, et al., Human norovirus culture in B cells. Nat Protoc, 2015. 10(12): p. 1939-47.

9. Robinson, C.M., P. R. Jesudhasan, and J.K. Pfeiffer, Bacterial lipopolysaccharide binding enhances virion stability and promotes environmental fitness of an enteric virus. Cell Host Microbe, 2014. 15(1): p. 36-46.

10. Baldridge, M. T., T. J. Nice, B. T. McCune, C. C. Yokoyama, A. Kambal, M. Wheadon, et al., Commensal microbes and interferon-lambda determine persistence of enteric murine norovirus infection. Science, 2015. 347 (6219): p. 266-9.

11. Sansone, C. L., J. Cohen, A. Yasunaga, J. Xu, G. Osborn, H. Subramanian, et al., Microbiota-Dependent Priming of Antiviral Intestinal Immunity in Drosophila. Cel1 Host Microbe, 2015. 18(5): p. 571-81.

12. Ichinohe, T., I. K. Pang, Y. Kumamoto, D. R. Peaper, J.H. Ho, T. S. Murray, et al., Microbiota regulates immune defense against respiratory tract influenza A virus infection. Proc Natl Acad Sci U S A, 2011. 108(13): p. 5354-9.

13. Ganal, S. C., S. L. Sanos, C. Kallfass, K. Oberle, C. Johner, C. Kirschning, et al., Priming of natural killer cells by nonmucosal mononuclear phagocytes requires instructive signals from commensal microbiota. Immunity, 2012. $37(1)$ : p. $171-86$.

14. Abt, M. C. and D. Artis, The dynamic influence of commensal bacteria on the immune response to pathogens. Curr Opin Microbiol, 2013. 16(1): p. 4-9.

15. Abt, M. C., L. C. Osborne, L.A. Monticelli, T. A. Doering, T. Alenghat, G.F. Sonnenberg, et al., Commensal bacteria calibrate the activation threshold of innate antiviral immunity. Immunity, 2012. 37(1): p. 158-70.

16. Steed, A. L., G. P. Christophi, G. E. Kaiko, L. Sun, V. M. Goodwin, U. Jain, et al., The microbial metabolite desaminotyrosine protects from influenza through type I interferon. Science, 2017. 357 (6350): p. 498-502.

17. Schaupp, L., S. Muth, L. Roge11, M. Kofoed-Branzk, F. Melchior, S. Lienenklaus, et al., Microbiota-Induced Type I Interferons Instruct a Poised Basal State of Dendritic Cells. Cel1, 2020. 181(5): p. 1080-1096 e19.

18. Winkler, E. S., S. Shrihari, B. L. Hykes, Jr., S. A. Handley, P. S. Andhey, Y.S. Huang, et al., The Intestinal Microbiome Restricts Alphavirus Infection and Dissemination through a Bile Acid-Type I IFN Signaling Axis. Cell, 2020. $182(4)$ : p. 901-918 e18.

19. Grau, K. R., S. Zhu, S. T. Peterson, E.W. Helm, D. Philip, M. Phillips, et al., The intestinal regionalization of acute norovirus infection is regulated by the microbiota via bile acid-mediated priming of type III interferon. Nat Microbiol, 2019.

20. Jones, M. K., M. Watanabe, S. Zhu, C. L. Graves, L. R. Keyes, K. R. Grau, et al., Enteric bacteria promote human and mouse norovirus infection of $B$ cells. Science, 2014. 346(6210): p. 755-9.

21. McCartney, S., W. Vermi, S. Gilfillan, M. Cella, T. L. Murphy, R. D. Schreiber, 
et al., Distinct and complementary functions of MDA5 and TLR3 in poly(I:C)mediated activation of mouse NK cells. J Exp Med, 2009. 206(13) : p. 2967-76.

22. Thackray, L. B., S. A. Handley, M. J. Gorman, S. Poddar, P. Bagadia, C. G. Briseno, et al., Oral Antibiotic Treatment of Mice Exacerbates the Disease Severity of Multiple Flavivirus Infections. Cell Rep, 2018. $22(13)$ : p. 3440-3453 e6.

23. Uchiyama, R., B. Chassaing, B. Zhang, and A. T. Gewirtz, Antibiotic treatment suppresses rotavirus infection and enhances specific humoral immunity. J Infect Dis, 2014. 210(2): p. 171-82.

24. Shi, Z., J. Zou, Z. Zhang, X. Zhao, J. Noriega, B. Zhang, et al., Segmented Filamentous Bacteria Prevent and Cure Rotavirus Infection. Ce11, 2019. 179(3): p. 644-658 e13.

25. Oberste, M. S., E. Gotuzzo, P. Blair, W. A. Nix, T. G. Ksiazek, J.A. Comer, et al., Human febrile illness caused by encephalomyocarditis virus infection, Peru. Emerg Infect Dis, 2009. 15(4): p. 640-6.

26. Nishimura, Y., M. Shimojima, Y. Tano, T. Miyamura, T. Wakita, and H. Shimizu, Human $P$-selectin glycoprotein ligand-1 is a functional receptor for enterovirus 71. Nat Med, 2009. 15(7): p. 794-7.

27. Erny, D., A. L. Hrabe de Angelis, D. Jaitin, P. Wieghofer, 0. Staszewski, E. David, et al., Host microbiota constantly control maturation and function of microglia in the CNS. Nat Neurosci, 2015. 18(7): p. 965-77.

28. Reizis, B., Plasmacytoid Dendritic Cells: Development, Regulation, and Function. Immunity, 2019. 50(1): p. 37-50.

29. Abreu, M. T., Toll-like receptor signalling in the intestinal epithelium: how bacterial recognition shapes intestinal function. Nat Rev Immunol, 2010. $10(2)$ : p. $131-44$.

30. Stefan, K. L., M. V. Kim, A. Iwasaki, and D. L. Kasper, Commensal Microbiota Modulation of Natural Resistance to Virus Infection. Ce11, 2020. 183(5): p. 1312-1324 e10.

31. Wang, G., S. Huang, Y. Wang, S. Cai, H. Yu, H. Liu, et a1., Bridging intestinal immunity and gut microbiota by metabolites. Cell Mol Life Sci, 2019. 76(20): p. $3917-3937$.

32. Antunes, K. H., J. L. Fachi, R. de Paula, E. F. da Silva, L. P. Pral, A. A. Dos Santos, et al., Microbiota-derived acetate protects against respiratory syncytial virus infection through a GPR43-type 1 interferon response. Nat Commun, 2019. 10(1): p. 3273.

33. Zhu, S., X. Ge, X. Gong, X. Guo, Y. Chen, and H. Yang, Alteration of encephalomyocarditis virus pathogenicity due to a mutation at position 100 of VP1. Sci China Life Sci, 2011. 54(6): p. 535-43.

34. Jia, H., X. Ge, X. Guo, H. Yang, K. Yu, Z. Chen, et al., Specific small interfering RNAs-mediated inhibition of replication of porcine encephalomyocarditis virus in BHK-21 cells. Antiviral Res, 2008. 79(2): p. 95-104.

35. Wang, P., S. Zhu, L. Yang, S. Cui, W. Pan, R. Jackson, et al., N1rp6 regulates intestinal antiviral innate immunity. Science, 2015. 350(6262): p. 826-30. 
781 Fig.1. Microbiota depletion deteriorates EMCV pathogenesis and viral replication in vivo. (A) Groups of 6-8 weeks old C57BL/6J mice were pretreated with PBS or Abx for 5 days, inoculated with different dose of EMCV orally and observed for 14 days. Survival curves $(n=12-17)$ and pathological scores $(n=10-15)$ were documented. (B) Blood and brain samples of PBS or Abx mice inoculated with $2 \times 10^{7} \mathrm{TCID}_{50}$ units of EMCV orally were harvested and tested for viral loads at 3 and 5 dpi by qPCR $(n=6)$. (C) Indirect fluorescent assay (IFA) of brain sections from PBS- or Abx-infected mice at 5 dpi. EMCV capsid protein VP2 and cellular surface markers were double-stained with respective antibodies (representative images from $n=6$ per group). (D) Groups of PBS, Abx or Abx mice received FMT from conventionally housed PBS naïve mice were intraperitoneally inoculated with $200 \mathrm{TCID}_{50}$ units of EMCV for survival kinetics analysis (n=13-17). (E) Viral titers of brain tissues collected from PBS-, Abx- or FMT-infected mice at 1 and 3 dpi (same dose and route as Fig. 1D, $n=5-6$ ). Data represented in the figures were from two independent experiments. Dotted dash lines indicate the limit of detection (LD).

Fig. 2. Innate cellular immune responses were severely diminished in Abx-treated mice post EMCV infection. (A) Frequency of a panel of innate immune cells including 797 inflammatory monocytes, NK cells, macrophages, pDC or cDC in PBMC or splenocytes of mock or infected PBS or Abx mice at 3 dpi $(n=4-8)$. (B) Frequency of various adaptive immune cells including $\mathrm{CD} 4^{+} \mathrm{T}$ cells, $\mathrm{CD} 8^{+} \mathrm{T}$ cells or $\mathrm{B}$ cells from PBMC or spleen of mock 
macrophages from mock- or infected-PBS or Abx mice at 3 dpi (upper two panels, histograms of MHC-I- or CD80-expressing macrophages; lower two panels, frequency of macrophages expressing MHC-I or CD80 in total PBMC macrophages; $n=6$ ). (D) Survival analysis of PBS or Abx mice treated with clodronate liposomes or control reagent following EMCV intraperitoneal inoculation $(n=10-11)$. (E) Blood viral titers of PBS or Abx mice treated with clodronate liposomes or control reagent at $1 \mathrm{dpi}(\mathrm{n}=6)$. Data presented in the figure were from two independent experiments.

Fig.3. Microbiota depletion results in significantly impaired systemic or cerebral type I IFN responses. Fold induction of Ifnb and ISG expression in PBMC (A) and spleen (B) of PBS or Abx mice at $12 \mathrm{hpi}$ following EMCV infection relative to respective mock controls $(n=5)$. (C) Expression of Ifnb and ISGs in the brain of infected PBS- or Abx-treated mice (foldchange compared to respective mock mice) at 1 or 3 dpi ( $n=5-6)$. IFN- $\beta$ levels in the brain at $3 \mathrm{dpi}$ as detected by ELISA (middle panel). (D) Fold induction of proinflammatory or immune regulatory cytokines expression in the brain at 1 or 3 dpi relative to respective

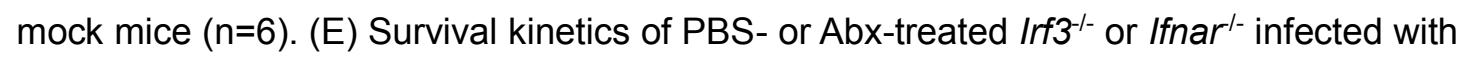
EMCV intraperitoneally, wild-type C57BL/6J mice were served as control (left panel, $n=10$ 12; middle panel, $n=9-14$; right panel, $n=13-15)$. ( $F$ ) Viral titers in blood and brain collected from infected PBS or Abx WT, Irf3-- or Ifnar ${ }^{-/}$mice with at $1 \mathrm{dpi}(\mathrm{n}=5-6)$. All data presented in the figure were from two independent experiments.

Fig.4. B. coccoides protects EMCV systemic infection by restricting viral in vivo replication. Viral titers (A) and induction of Ifnb (B) in the brain of PBS-, Abx- or single antibiotic-treated mice were measured by qPCR at 3 dpi $(n=5-6)$. (C) Bacterial richness was defined by the 
number of the unique taxa. (D) Heat map of relative abundance from different groups of antibiotic treatment. (E) Relative abundance changes of bacteria at genus level. (F) Survival analysis of Abx mice colonized with B. coccoides (B.C), A. muciniphila (AKK), L. reuteri (L.R), C. butyricum (C.B), or mouse fecal contents (FMT) following EMCV intraperitoneal inoculation (3 experiments, $n=16-20$ ). (G) Viral burden in brain tissues harvested from mock- or infected-PBS, Abx or Abx mice colonized with FMT, B.C or C.B at $3 \mathrm{dpi}(\mathrm{n}=6)$. All data presented in the figure were from at least two independent experiments.

Fig.5. B. coccoides colonization restricts enteric virus systemic infection by activating innate cellular immune responses and type I IFN expression in a macrophage- and Ifnardependent manner. Frequency of macrophages expressing MHC-I (A) or CD80 (B) in PBMC or spleen isolated from PBS, Abx or Abx mice colonized with FMT, B.C or C.B at 3 dpi following EMCV inoculation (representative flow cytometry histograms of showing surface staining of MHC-I or CD80, n=6). (C) Survival kinetics of infected Abx and B.Ccolonized Abx mice injected with clodronate liposomes ( $n=9-10)$. (D) Expression level of Ifnb in PBMC isolated from infected PBS, Abx, or Abx mice gavaged with FMT, B.C or C.B, comparing to their respective mock controls ( $n=5)$. (E) Survival kinetics of infected Abx and B.C-colonized Abx Ifnar'-mice $(n=6-9)$. (F) Viral titers in blood and brain collected from infected PBS, Abx or B.C-colonized WT or Ifnar ${ }^{/-}$mice at $1 \mathrm{dpi}(\mathrm{n}=5-6)$. All data presented in the figure were from two independent experiments.

Fig.6. B. coccoides colonization promotes type I IFN and ISG responses in macrophages to limit EMCV infection. (A) Ifnb and antiviral defense gene expression in BMDMs isolated from PBS, Abx, B.C- or C.B-colonized Abx mice at $8 \mathrm{~h}$ post EMCV infection (MOI=5) in 
vitro ( $\mathrm{n}=5-6)$. (B) Relative mRNA expression of Oas1a and Mx1 in BMDMs isolated from WT or Ifnar/- mice that pretreated with PBS, Abx or Abx plus B. coccoides colonization at 8 hpi in vitro (n=5-6). (C) Splenic macrophages sorted from spleen of PBS, Abx or B.C-

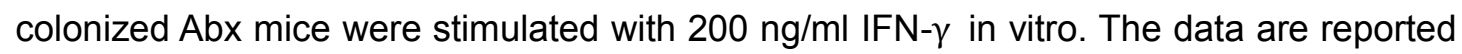
as the averaged frequency of pSTAT1-stained CD11 $\mathrm{b}^{+}$cell subsets at $16 \mathrm{~h}$ subtracted by the frequency of these cells tested at $0 \mathrm{~h}$ post IFN- $\gamma$ stimulation (3 experiments, $\mathrm{n}=6)$. (D) Ifnb and antiviral defense gene expression in splenic macrophages isolated from EMCVinfected PBS, Abx, or B.C-colonized Abx mice at 1 dpi $(n=6)$. (E) Relative mRNA expression of $\mathrm{Cxcl} 10$ and Stat2 in splenic macrophages isolated from EMCV-infected WT or Ifnar/- mice that pretreated with PBS, Abx or Abx plus B. coccoides colonization at $1 \mathrm{dpi}$ $(n=5-6)$. Data presented in the figure were from at least two independent experiments.

Fig. S1. Flow cytometry gating strategy of NK cells in PBMC of mock or infected mice w/wo Abx treatment at 3 dpi (related to Fig. 2). Representative flow cytometry plots showing gating scheme for NK1.1-PE positive cells in PBMC isolated from PBS mock, Abx mock, PBS-infected or Abx-infected mice.

Fig. S2. Flow cytometry gating strategy of macrophages in PBMC of mock or infected mice w/wo Abx treatment at 3 dpi (related to Fig. 2). Representative flow cytometry plots showing gating scheme for F4/80-CD11b double-positive cell subsets in PBMC isolated from PBS mock mice and representative histogram image of $\mathrm{MHC}-\mathrm{I}^{\mathrm{hi}}(\mathrm{A})$ or $\mathrm{CD} 80(\mathrm{~B})$ staining on the surface of these cell subsets (left, lower panel); same gating strategy is utilized for all mice groups and the histogram overlay images are presented (right, lower panel).

Fig. S3. Depletion of splenic macrophages following administration of clodronate 

mice treated with control reagents $(A)$ or clodronate liposomes $(B)$.

Fig. S4. Relative abundance of bacteria strains in fecal samples collected from single bacterium colonized-mice. Groups of Abx mice were colonized with B. coccoides (A) or $C$.

871 butyricum (B) and fecal relative bacterial abundance at $48 \mathrm{~h}$ post colonization was 872 determined by $16 \mathrm{~S}$ rRNA sequencing $(n=6)$.

873 Fig. S5. Flow cytometry gating strategy of sorted splenic macrophages from PBS, Abx or 874 B.C-colonized Abx mice that stained for pSTAT1 post IFN- $\gamma$ stimulation in vitro (related to 875 Fig.6). Representative flow cytometry plots showing sorting scheme for F4/80-CD11b 876 double-positive cell subsets of splenocytes isolated from PBS mice (upper panels) and 877 representative histogram image of intracellular staining of pSTAT1 in sorted macrophages 878 at $0 \mathrm{~h}$ post IFN- $\gamma$ stimulation (left, lower panel); same gating strategy is utilized for pSTAT1 879 staining of sorted splenic macrophages from PBS, Abx or B.C-colonized Abx mice and the 880 histogram overlay images of pSTAT1 staining at $16 \mathrm{~h}$ post IFN- $\gamma$ stimulation are presented 881 (right, lower panel). 
A
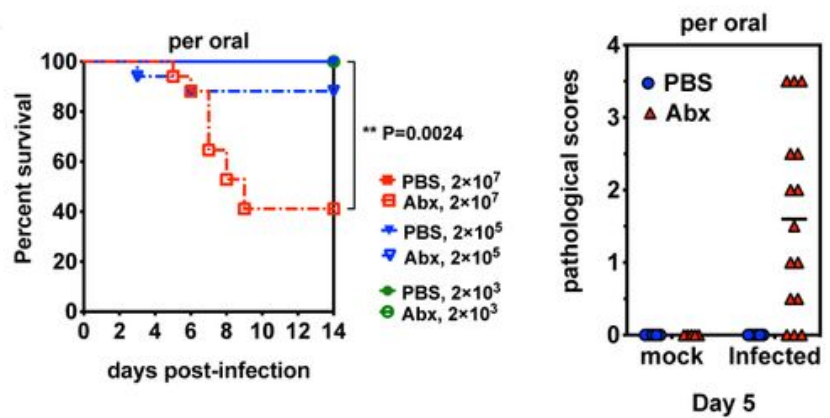

B
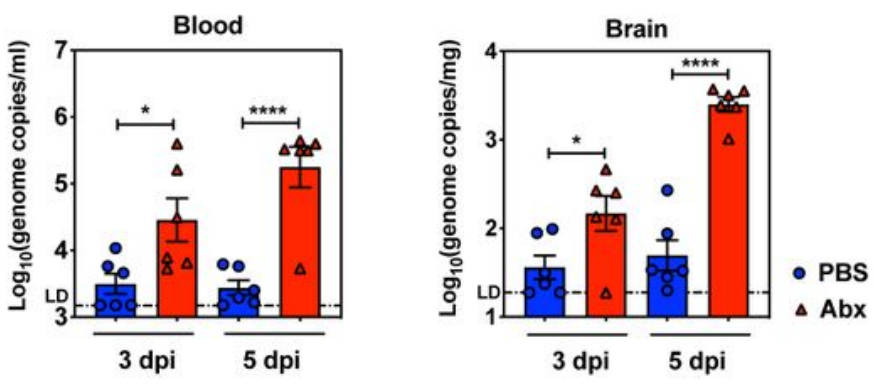

C
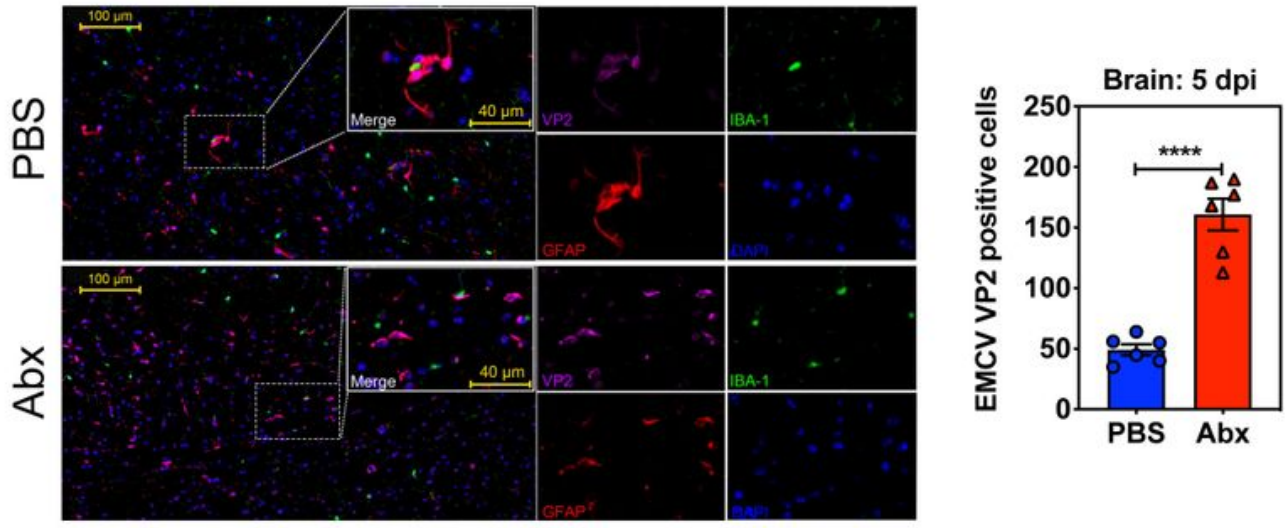

D

E
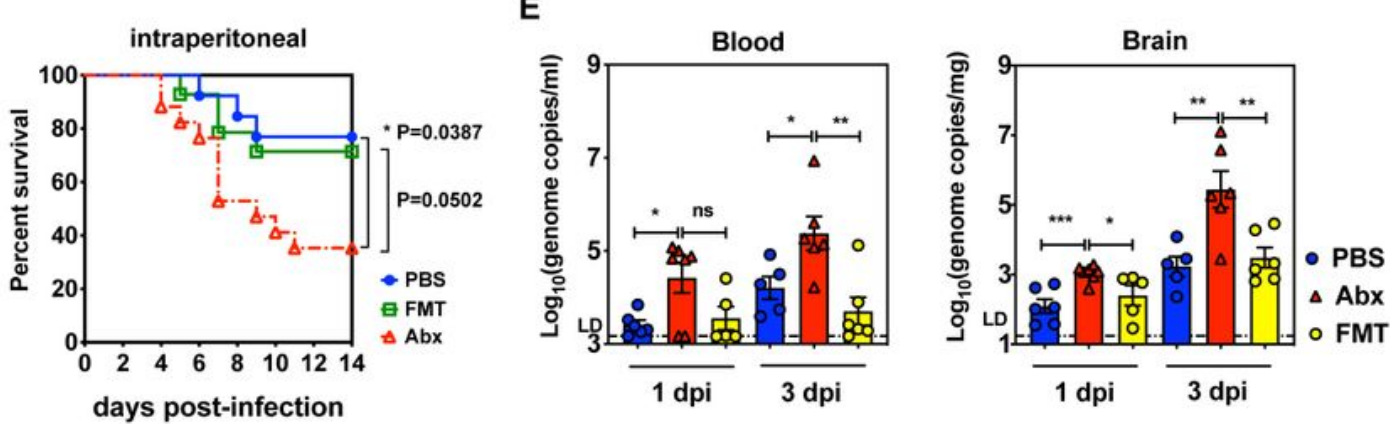

\section{Figure 1}

Microbiota depletion deteriorates EMCV pathogenesis and viral replication in vivo. (A) Groups of 6-8 weeks old C57BL/6J mice were pretreated with PBS or Abx for 5 days, inoculated with different dose of EMCV orally and observed for 14 days. Survival curves $(n=12-17)$ and pathological scores $(n=10-15)$ were 
documented. (B) Blood and brain samples of PBS or Abx mice inoculated with $2 \times 10^{7} \mathrm{TCID}_{50}$ units of EMCV orally were harvested and tested for viral loads at 3 and 5 dpi by qPCR $(n=6)$. (C) Indirect fluorescent assay (IFA) of brain sections from PBS- or Abx-infected mice at $5 \mathrm{dpi}$. EMCV capsid protein VP2 and cellular surface markers were double-stained with respective antibodies (representative images from $n=6$ per group). (D) Groups of PBS, Abx or Abx mice received FMT from conventionally housed PBS naïve mice were intraperitoneally inoculated with $200 \mathrm{TCID}_{50}$ units of EMCV for survival kinetics analysis $(n=13-17)$. (E) Viral titers of brain tissues collected from PBS-, Abx- or FMT-infected mice at 1 and 3 dpi (same dose and route as Fig. 1D, n=5-6). Data represented in the figures were from two independent experiments. Dotted dash lines indicate the limit of detection (LD). 
A

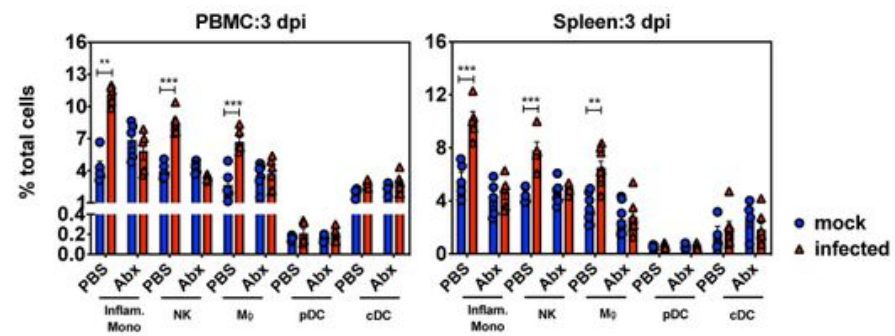

B

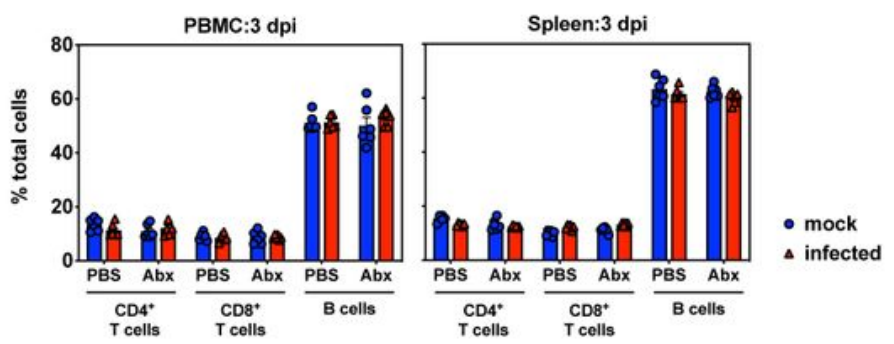

C
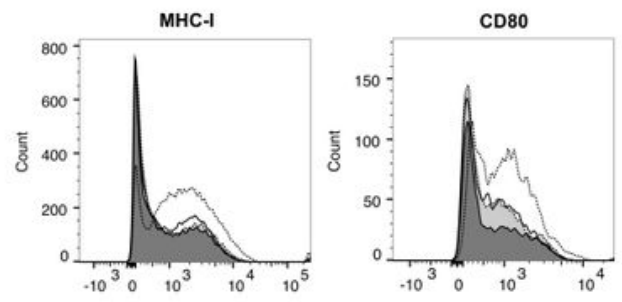

......... PBS $\ldots . . . . .$. PBS infected
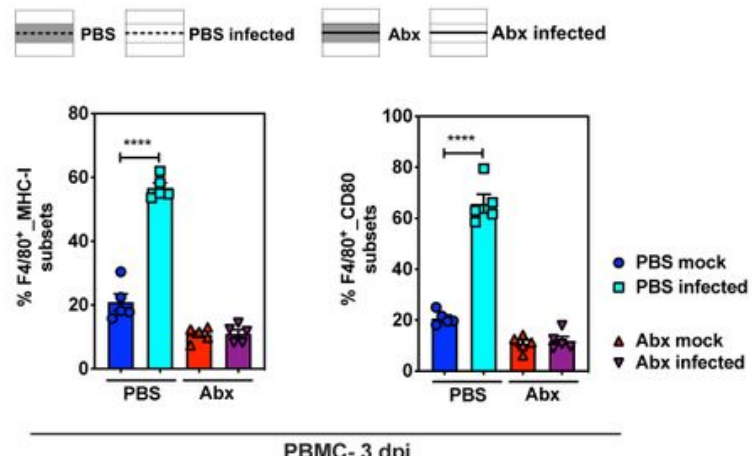

D

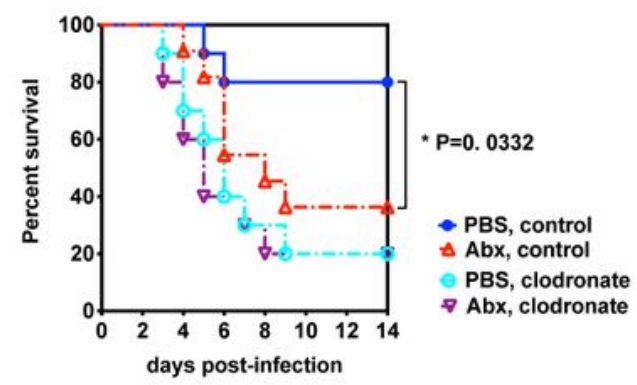

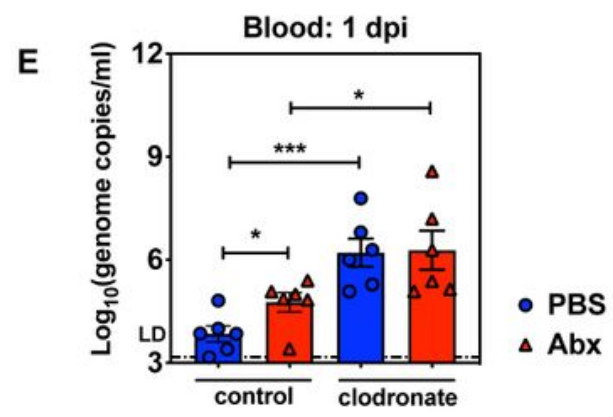

\section{Figure 2}

Innate cellular immune responses were severely diminished in Abx-treated mice post EMCV infection. (A) Frequency of a panel of innate immune cells including inflammatory monocytes, NK cells, macrophages, $\mathrm{pDC}$ or $\mathrm{CDC}$ in PBMC or splenocytes of mock or infected PBS or Abx mice at $3 \mathrm{dpi}(\mathrm{n}=4-8)$. (B) Frequency of various adaptive immune cells including CD4+ T cells, CD8+ T cells or B cells from PBMC or spleen of mock or infected PBS or Abx mice at $3 \mathrm{dpi}(\mathrm{n}=6)$. (C) Expression of MHC-I and CD80 on PBMC 
macrophages from mock- or infected-PBS or Abx mice at $3 \mathrm{dpi}$ (upper two panels, histograms of MHC-Ior CD80-expressing macrophages; lower two panels, frequency of macrophages expressing MHC-I or CD80 in total PBMC macrophages; $n=6$ ). (D) Survival analysis of PBS or Abx mice treated with clodronate liposomes or control reagent following EMCV intraperitoneal inoculation ( $n=10-11)$. (E) Blood viral titers of PBS or Abx mice treated with clodronate liposomes or control reagent at $1 \mathrm{dpi}(n=6)$. Data presented in the figure were from two independent experiments.
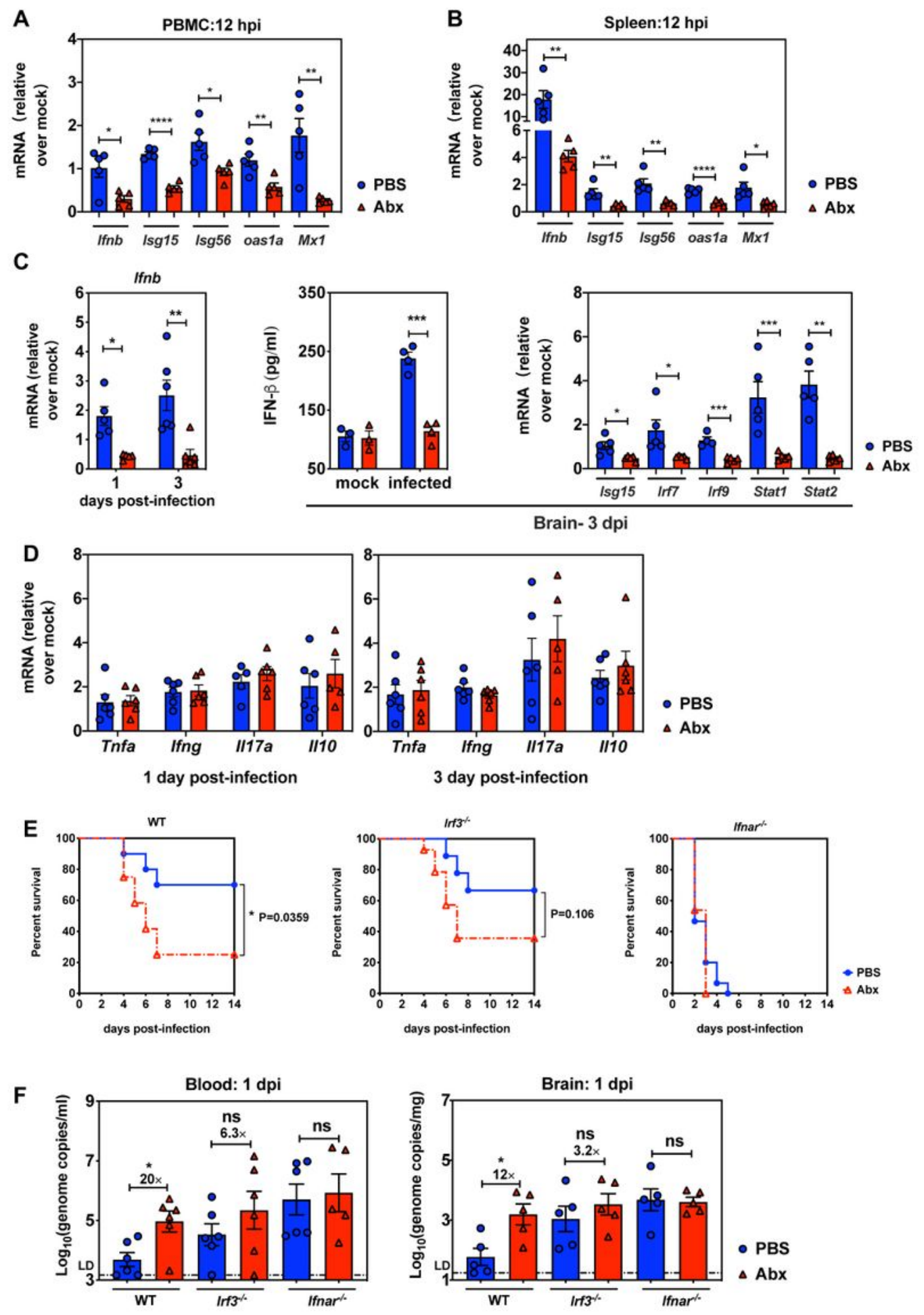

Figure 3 
Microbiota depletion results in significantly impaired systemic or cerebral type I IFN responses. Fold induction of Ifnb and ISG expression in PBMC (A) and spleen (B) of PBS or Abx mice at 12 hpi following EMCV infection relative to respective mock controls $(n=5)$. (C) Expression of Ifnb and ISGs in the brain of infected PBS- or Abx-treated mice (fold-change compared to respective mock mice) at 1 or $3 \mathrm{dpi}(\mathrm{n}=5-6)$. IFN- $\beta$ levels in the brain at $3 \mathrm{dpi}$ as detected by ELISA (middle panel). (D) Fold induction of proinflammatory or immune regulatory cytokines expression in the brain at 1 or 3 dpi relative to respective mock mice $(n=6)$. (E) Survival kinetics of PBS- or Abx-treated Irf3-/- or Ifnar-/- infected with EMCV intraperitoneally, wild-type C57BL/6J mice were served as control (left panel, $n=10-12$; middle panel, $n=9-14$; right panel, $n=13-15)$. (F) Viral titers in blood and brain collected from infected PBS or Abx WT, Irf3-/- or Ifnar-/- mice with at $1 \mathrm{dpi}(\mathrm{n}=5-6)$. All data presented in the figure were from two independent experiments. 
A

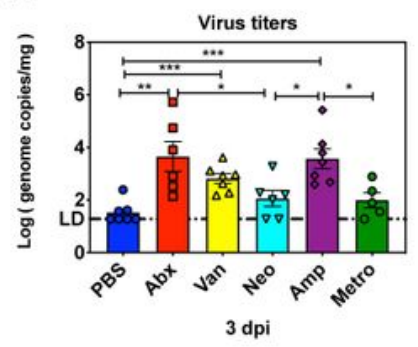

B

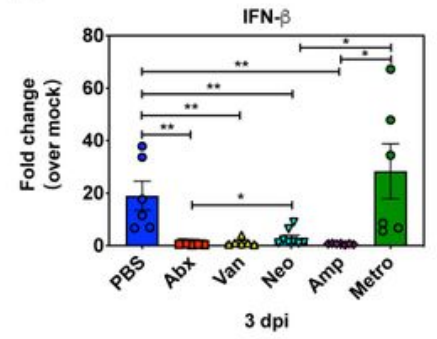

C

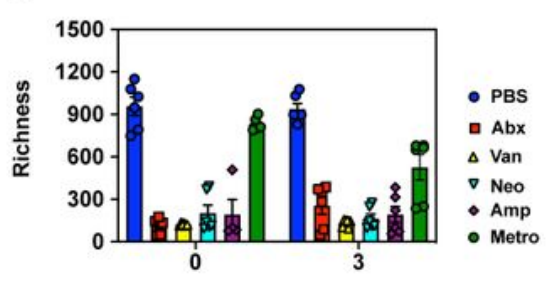

D

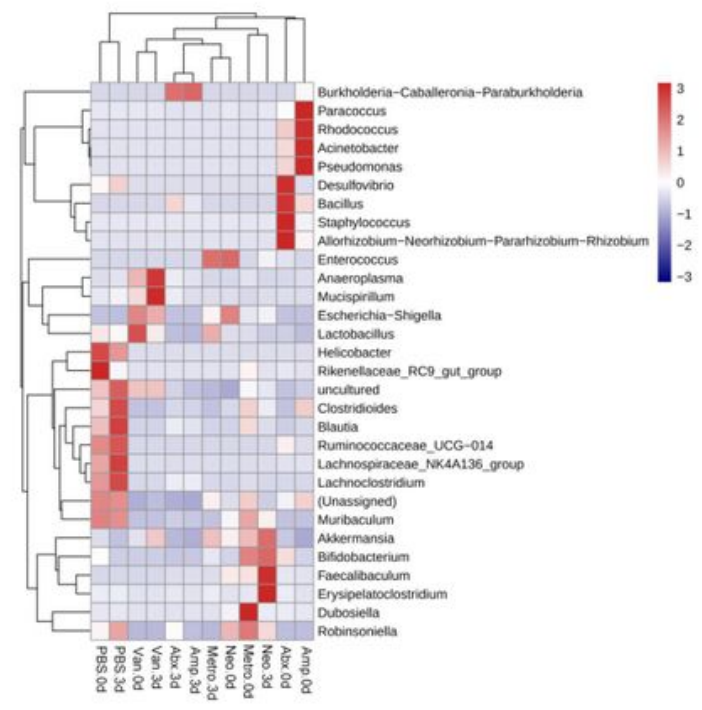

E
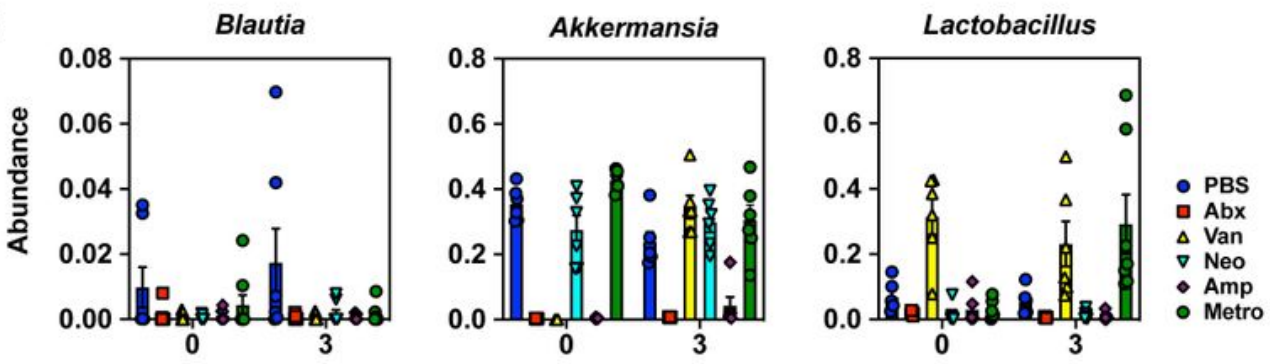

F
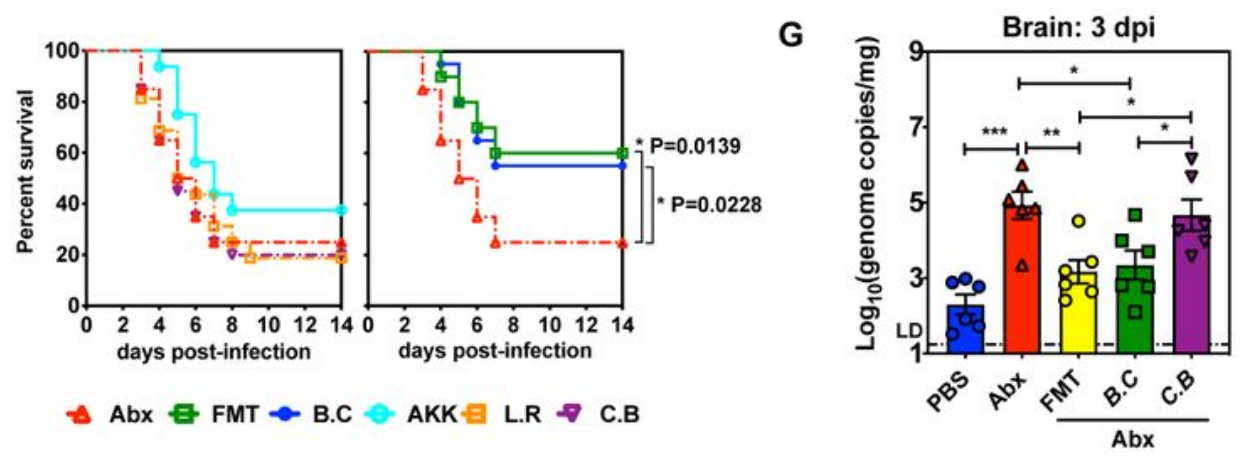

\section{Figure 4}

B. coccoides protects EMCV systemic infection by restricting viral in vivo replication. Viral titers $(A)$ and induction of Ifnb (B) in the brain of PBS-, Abx- or single antibiotic-treated mice were measured by qPCR at 3 dpi (n=5-6). (C) Bacterial richness was defined by the number of the unique taxa. (D) Heat map of relative abundance from different groups of antibiotic treatment. (E) Relative abundance changes of bacteria at genus level. (F) Survival analysis of Abx mice colonized with B. coccoides (B.C), A. 
muciniphila (AKK), L. reuteri (L.R), C. butyricum (C.B), or mouse fecal contents (FMT) following EMCV intraperitoneal inoculation (3 experiments, $n=16-20)$. (G) Viral burden in brain tissues harvested from mock- or infected-PBS, Abx or Abx mice colonized with FMT, B.C or C.B at $3 \mathrm{dpi}(n=6)$. All data presented in the figure were from at least two independent experiments.
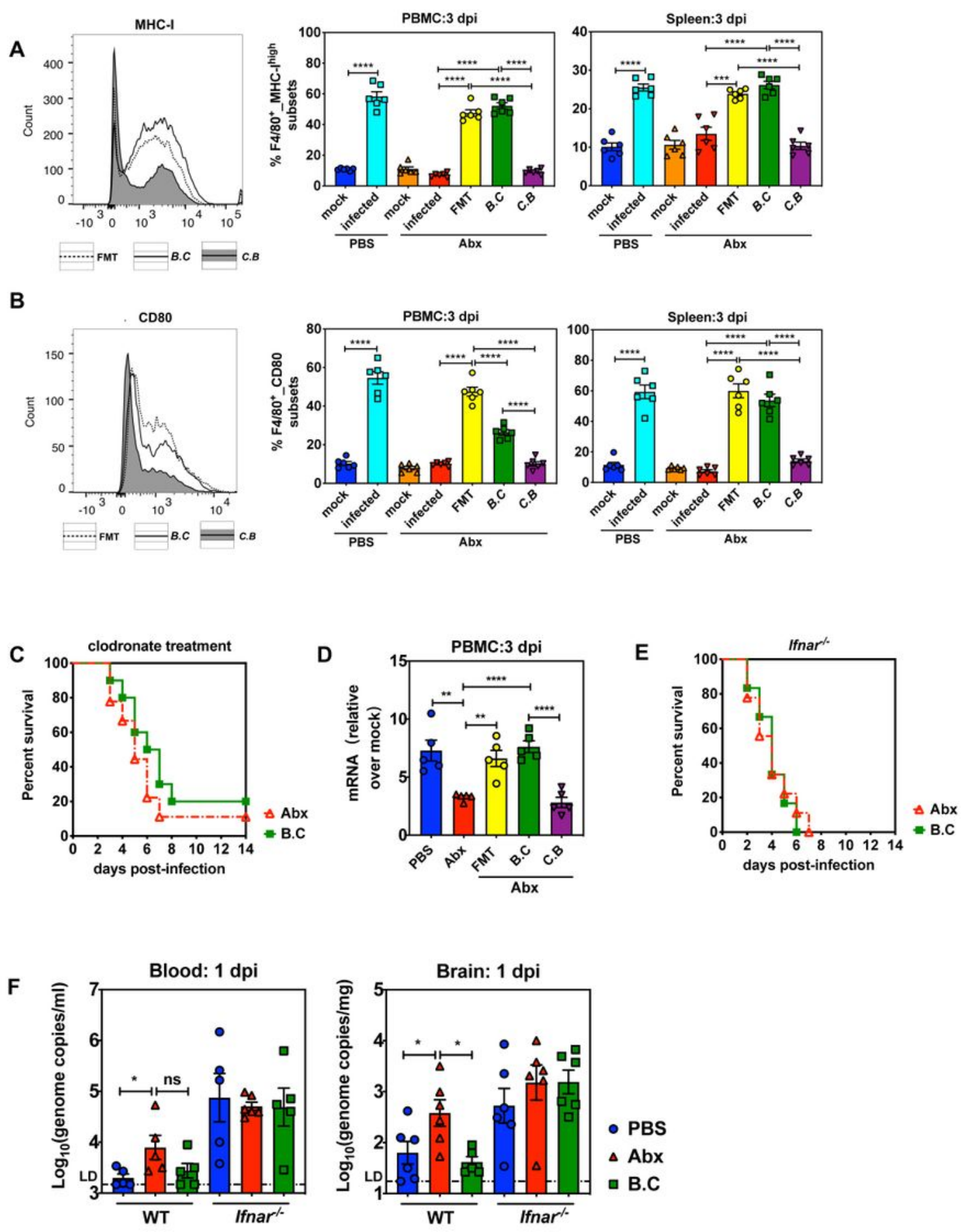

Figure 5 
B. coccoides colonization restricts enteric virus systemic infection by activating innate cellular immune responses and type I IFN expression in a macrophage- and Ifnar-dependent manner. Frequency of macrophages expressing MHC-I (A) or CD80 (B) in PBMC or spleen isolated from PBS, Abx or Abx mice colonized with FMT, B.C or C.B at 3 dpi following EMCV inoculation (representative flow cytometry histograms of showing surface staining of $M H C-I$ or $C D 80, n=6$ ). (C) Survival kinetics of infected $A b x$ and B.C-colonized Abx mice injected with clodronate liposomes ( $n=9-10)$. (D) Expression level of Ifnb in PBMC isolated from infected PBS, Abx, or Abx mice gavaged with FMT, B.C or C.B, comparing to their respective mock controls $(n=5)$. (E) Survival kinetics of infected Abx and B.C-colonized Abx Ifnar-/-mice $(n=6-9)$. (F) Viral titers in blood and brain collected from infected PBS, Abx or B.C-colonized WT or Ifnar-/- mice at 1 dpi $(n=5-6)$. All data presented in the figure were from two independent experiments. 
A
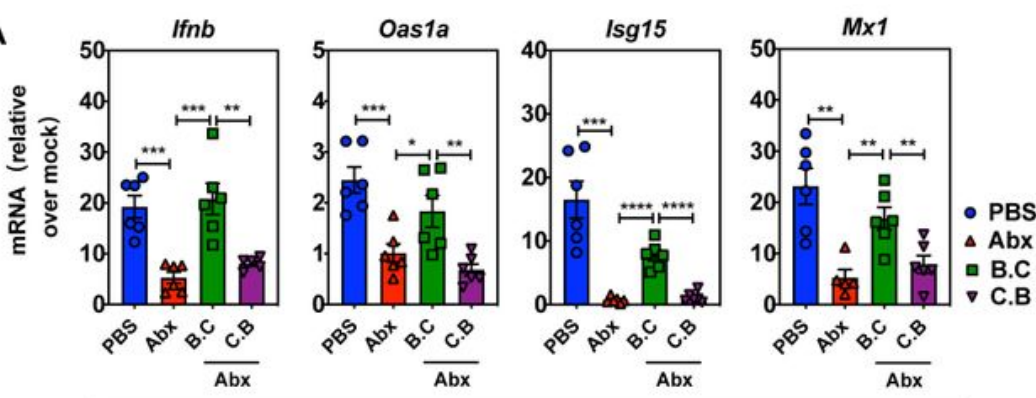

BMDM- 8 hpi

B

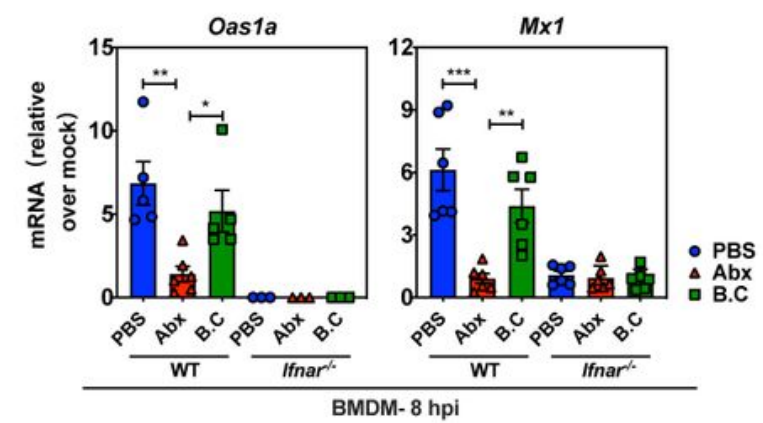

C

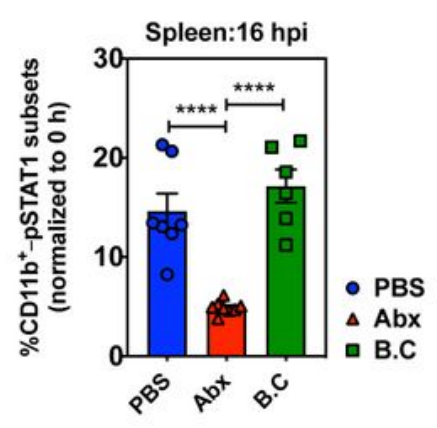

D

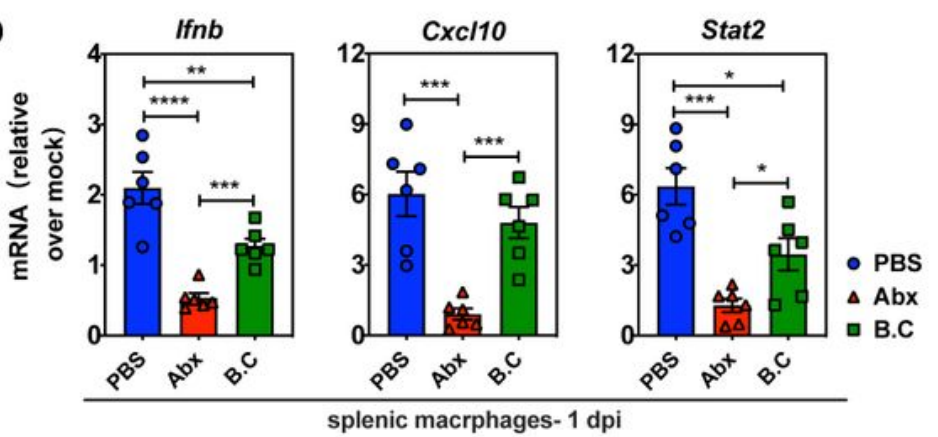

E

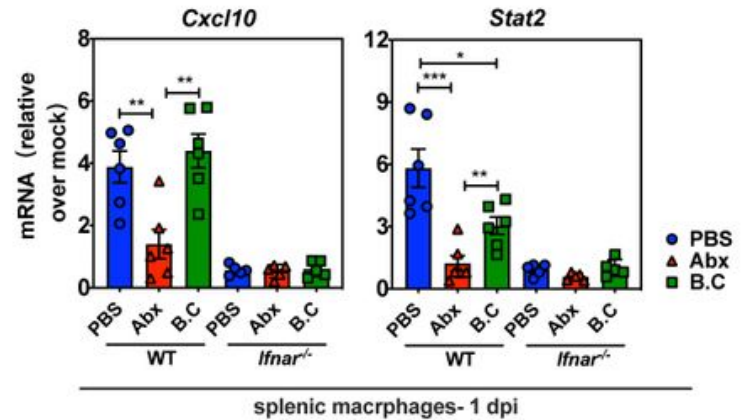

Figure 6

B. coccoides colonization promotes type I IFN and ISG responses in macrophages to limit EMCV infection. (A) Ifnb and antiviral defense gene expression in BMDMs isolated from PBS, Abx, B.C- or C.Bcolonized $\mathrm{Abx}$ mice at $8 \mathrm{~h}$ post EMCV infection (MOI=5) in vitro $(n=5-6)$. (B) Relative mRNA expression of Oas1a and Mx1 in BMDMs isolated from WT or Ifnar-/- mice that pretreated with PBS, Abx or Abx plus B. coccoides colonization at $8 \mathrm{hpi}$ in vitro $(n=5-6)$. (C) Splenic macrophages sorted from spleen of PBS, Abx 
or B.C-colonized Abx mice were stimulated with $200 \mathrm{ng} / \mathrm{ml} \mathrm{IFN- \gamma}$ in vitro. The data are reported as the averaged frequency of pSTAT1-stained CD11b+ cell subsets at $16 \mathrm{~h}$ subtracted by the frequency of these cells tested at $0 \mathrm{~h}$ post IFN- $y$ stimulation (3 experiments, $n=6$ ). (D) Ifnb and antiviral defense gene expression in splenic macrophages isolated from EMCV-infected PBS, Abx, or B.C-colonized Abx mice at $1 \mathrm{dpi}(\mathrm{n}=6)$. (E) Relative mRNA expression of $\mathrm{Cxcl} 10$ and Stat2 in splenic macrophages isolated from EMCV-infected WT or Ifnar-/- mice that pretreated with PBS, Abx or Abx plus B. coccoides colonization at $1 \mathrm{dpi}(\mathrm{n}=5-6)$. Data presented in the figure were from at least two independent experiments.

\section{Supplementary Files}

This is a list of supplementary files associated with this preprint. Click to download.

- Supplementaryfigures.pdf

- TableS1.docx 